\title{
A search for planets in the metal-enriched binary HD 219542 ${ }^{\star}$
}

\author{
S. Desidera ${ }^{1}$, R. G. Gratton ${ }^{1}$, M. Endl ${ }^{2}$, M. Barbieri ${ }^{3}$, R. U. Claudi ${ }^{1}$, R. Cosentino ${ }^{4,5}$, S. Lucatello ${ }^{1,6}$, \\ F. Marzari ${ }^{7}$, and S. Scuderi ${ }^{3}$
}

1 INAF - Osservatorio Astronomico di Padova, Vicolo dell' Osservatorio 5, 35122 Padova, Italy e-mail: gratton, claudi, lucatello@pd.astro.it

2 McDonald Observatory, The University of Texas at Austin, Austin, TX 78712, USA

e-mail: mike@astro.as.utexas.edu

3 CISAS, c/o Dipartimento di Fisica, Università di Padova, via Marzolo 8, 35131 Padova, Italy e-mail: mauro.barbieri@pd.infn.it

${ }^{4}$ INAF - Oss. Astrofisico di Catania, via S. Sofia 78, Catania, Italy e-mail: scuderi@ct.astro.it

5 INAF - Centro Galileo Galilei, calle Alvarez de Abreu 70, 38700 Santa Cruz de La Palma (TF), Spain e-mail: cosentino@tng.iac.es

${ }^{6}$ Dipartimento di Astronomia, Universitá di Padova, Vicolo dell'Osservatorio 2, Padova, Italy

7 Dipartimento di Fisica, Universitá di Padova, via Marzolo 8, 35131 Padova, Italy

e-mail: francesco.marzari@pd.infn.it

Received 3 Febraury 2003 / Accepted 21 March 2003

\begin{abstract}
The components of the wide binary HD 219542 were recently found to differ in metallicity by about 0.1 dex (Gratton et al. 2001b). In this paper, we present the results of 2 years of high precision radial velocity monitoring of these stars performed at the Telecopio Nazionale Galileo (TNG) using the high resolution spectrograph SARG. No indication for radial velocity variations above the measurement errors $\left(\sim 5 \mathrm{~m} \mathrm{~s}^{-1}\right)$ was found for the metal richer component $\mathrm{A}$. This allows us to place upper mass-limits for planets around this star. HD 219542 B instead shows a low amplitude variation with a 112 day period at a confidence level of $\sim 96-97 \%$. This might suggest the presence of a Saturn-mass planet, although it is still possible that these variations are due to moderate activity of the star. Tests based on variations of bisectors, stellar magnitude and line equivalent widths were inconclusive so far.
\end{abstract}

Key words. stars: planetary systems - stars: binaries: visual - stars: individual: HD 219542 - stars: activity techniques: radial velocities - techniques: spectroscopic

\section{Introduction}

The increasing number of extrasolar planets discovered by radial velocity surveys (over 100, see e.g. Butler et al. 2002; Butler et al. 2003; Udry et al. 2002) reveals a surprising variety of planetary systems: giant planets very close to their parent stars, planets in very eccentric orbits, multiple planets systems, in some cases showing orbital resonances. Further dedicated efforts are required for a detailed understanding of frequency and properties of giant planets as a function of some

Send offprint requests to: S. Desidera,

e-mail: desidera@pd.astro.it

* Based on observations made with the Italian Telescopio Nazionale Galileo (TNG) operated on the island of La Palma by the Centro Galileo Galilei of the INAF (Istituto Nazionale di Astrofisica) at the Spanish Observatorio del Roque de los Muchachos of the Instituto de Astrofisica de Canarias, and observations collected at the European Southern Observatory, Chile, using FEROS spectrograph at $1.5 \mathrm{~m}$ telescope (proposal ID: 69.D-0338). fundamental parameters (mass, chemical composition of parent star, dynamical environment).

More than half of the stars in the solar neighborhood are in binary systems. Recent discoveries of planets in binary systems with different mass ratios and separations indicate that planets might form in binaries. The case of 16 Cyg B is remarkable, showing radial velocity variations due to a massive planet orbiting the star at $1.7 \mathrm{AU}$ (Cochran et al. 1997). The very high eccentricity $(e=0.68)$ might be induced by dynamical perturbations of 16 Cyg A (Holman et al. 1997; Mazeh et al. 1997). Even more compelling is the case of $\gamma$ Cep, that harbours a planet in spite of the presence of a low-mass stellar companion in a 70-year orbit (Hatzes et al. 2003). Zucker \& Mazeh (2002) noted that planets found in binaries have a different mass-period correlation than planets orbiting single stars, with an overabundance of short-period high-mass planets.

Besides the study of dynamical effects (minimum binary separation for planets formation, perturbations of planetary 
orbits), binary systems could be used to explore the origin of the connection between high metallicity and occurrence of planetary companions (Gonzalez et al. 2001; Santos et al. 2001). Two possible explanations were suggested: either a high metal content makes it easier to form planets (and in this case no metallicity difference is expected between members of a binary system with and without planetary companions) or the high metallicity is the result of planets or planetesimal ingestion (Gonzalez 1997). It is also possible that both explanations are valid.

Infall of some planetary material on the star surface is expected during the formation and subsequent evolution of a planetary system. This may happen during the early phases of the planet formation, or in a more mature stage of the planetary system evolution, with the infall of remnant of the protoplanetary disk or with the ingestion of already formed planets, scattered toward the star by dynamical interactions with other planets in the system, distant stellar companions or passing stars.

If the material engulfed by the star is depleted in hydrogen and helium, the chemical composition of the star can be modified, leading to metal rich convective envelopes. This alteration is small or negligible for stars in the pre main sequence phase, that have thick convective zones. Stellar pollution is likely efficient mostly for main sequence stars with masses $1.0-1.3 M_{\odot}$, that have a convective envelope thin enough to allow a significant metallicity enhancement for reasonable quantities of accreted material.

The search for stellar pollution signatures was prompted by the evidence that the host stars of the extrasolar planets found by radial velocity surveys are typically metal-rich. The stars with planets were searched for trends in metallicity as a function of stellar mass or size of convective envelope (Santos et al. 2001; Pinsonneault et al. 2001), for anomalous abundance ratios, signatures of accretion of chemically fractionated material (Smith et al. 2001), and for lithium content anomalies (Ryan 2000; Israelian et al. 2001; Reddy et al. 2002). Lithium, and particularly the ${ }^{6} \mathrm{Li}$ isotope is in fact a good diagnostic for accretion since it is destroyed during the stellar lifetime. Murray et al. (2001) studied instead field main sequence stars with spectroscopically determined iron abundance. They found evidences for typical accretion of $0.5 M_{\oplus}$ of iron during the main sequence lifetime.

However, these results are hardly conclusive because of the lack of a suitable reference representing the original, unpolluted abundances.

Wide binaries represent instead easier targets to search for chemical anomalies, because of the comparison immediately provided by the two companions. Furthermore, their physical association (i.e. same distance) allows the use of differential analysis between the components. In fact, some of the most compelling results on the chemical anomalies of the stars with planets were achieved by studying the stars with planets member of binary systems e.g. 16 Cyg (King et al. 1997; Laws \& Gonzalez 2001) and HD 178911 (Zucker et al. 2002).

Differential techniques for the determination of stellar abundances are very sensitive for pair of stars with similar temperatures and gravities, i.e. main sequence stars with similar masses. This argument was at the basis of our project for the study of a sample of wide binaries, aimed to search for planets (using radial velocities) and the chemical composition signatures due to the engulfment of planetary material. This survey is on-going at TNG (the Italian Telescopio Nazionale Galileo, INAF, on La Palma, Canary Islands, Spain), using the high resolution spectrograph SARG (Gratton et al. 2001a).

The first result of our survey was the discovery of a pair (HD 219542) whose components differ in iron content by about 0.1 dex (Gratton et al. 2001b, hereinafter Paper I), with hints that the overabundance of the primary is confined to rocky elements, while volatiles have comparable abundances. The primary is also lithium rich. The most likely explanation of this abundance pattern is the infall of planetary material on the primary, either portions or remnants of a circumstellar disk or already formed planets. Reddy et al. (2002) searched for ${ }^{6} \mathrm{Li}$ in HD $219542 \mathrm{~A}$. This isotope represents a very powerful trace of recent accretion. They find ${ }^{6} \mathrm{Li} /{ }^{7} \mathrm{Li}=0.03 \pm 0.03$, clearly not conclusive. It must be noted that such an amount of ${ }^{6} \mathrm{Li}$ is nearly the one expected on the basis of the observed iron difference.

In this paper, we present the results of the 2 years radial velocity monitoring of the components of HD 219542 aimed at finding planets around them. In this case, we are searching for planets around stars showing evidence of accretion of planetary material. This effort is complementary to the searches for abundance anomalies of stars around which planets have already been detected, and may represent an important piece in the understanding of the origin of the observed chemical composition differences.

The paper is organized as follows: in Sects. 2 and 3 the available information concerning the stellar and orbital characteristics of the HD 219542 binary system are described; in Sect. 4 our high precision radial velocity measurements are presented; in Sect. 5 the significance of the variability of HD 219542 B is discussed; in Sect. 6 we discuss the possibility that the observed variations are due to activity-related phenomena; in Sect. 7 upper limits to the planets still allowed to exist around HD 219542 A are derived; in Sect. 8 the implications of our result are discussed.

\section{Stellar properties}

HD 219542 (HIP 114914, BD -02 5917, ADS 16642) is a wide pair of main sequence solar type stars. The physical associations of the components was confirmed by the Hipparcos astrometry. The main properties of the components of HD 219542 are summarized in Table 1.

\subsection{Stellar masses and ages}

Isochrone fitting can only poorly constrain the age of unevolved main sequence stars. The position in the color-magnitude diagram is best fitted by an age in the range 1-2 Gyr. The other indirect age indicators like chromosperic activity, $\mathrm{X}$-ray emission, lithium, and rotational velocity are discussed in Sects. 2.2-2.4.

The stellar masses derived using the $Z=0.031$ and $2 \mathrm{Gyr}$ isochrones of Girardi et al. (2000) are 1.15-1.12 and 1.07-1.05 
Table 1. Stellar properties of the components of HD 219542.

\begin{tabular}{|c|c|c|c|}
\hline Parameter & HD $219542 \mathrm{~A}$ & HD $219542 \mathrm{~B}$ & Ref. \\
\hline$\alpha(2000)$ & 231635.24 & 231635.42 & 1 \\
\hline$\delta(2000)$ & -013511.5 & -013507.0 & 1 \\
\hline$\mu_{\alpha}\left(\operatorname{mas} \mathrm{yr}^{-1}\right)$ & $163.28 \pm 2.12$ & $163.28 \pm 2.12$ & 1 \\
\hline$\mu_{\delta}\left(\operatorname{mas} \mathrm{yr}^{-1}\right)$ & $20.97 \pm 1.52$ & $20.97 \pm 1.52$ & 1 \\
\hline$R V\left(\mathrm{~km} \mathrm{~s}^{-1}\right)$ & $-11.1 \pm 0.2$ & $-10.0 \pm 0.2$ & 2 \\
\hline$\pi$ (mas) & \multicolumn{2}{|c|}{$18.30 \pm 1.97$} & 1 \\
\hline$d(\mathrm{pc})$ & \multicolumn{2}{|c|}{$54.6 \pm 5.9$} & 1 \\
\hline$U\left(\mathrm{~km} \mathrm{~s}^{-1}\right)$ & \multicolumn{2}{|c|}{$-40.5 \pm 4.8$} & 2 \\
\hline$V\left(\mathrm{~km} \mathrm{~s}^{-1}\right)$ & \multicolumn{2}{|c|}{$-16.4 \pm 3.6$} & 2 \\
\hline$W\left(\mathrm{~km} \mathrm{~s}^{-1}\right)$ & \multicolumn{2}{|c|}{$-4.0 \pm 2.5$} & 2 \\
\hline$V_{T}$ & $8.172 \pm 0.019$ & $8.591 \pm 0.028$ & 3 \\
\hline$(B-V)_{T}$ & $0.705 \pm 0.029$ & $0.791 \pm 0.044$ & 3 \\
\hline$V$ & $8.198 \pm 0.021$ & $8.589 \pm 0.021$ & 4 \\
\hline$V-I$ & $0.641 \pm 0.035$ & $0.705 \pm 0.037$ & 4 \\
\hline$H_{\mathrm{p}}$ & $8.312 \pm 0.006$ & $8.689 \pm 0.008$ & 1 \\
\hline$H_{\mathrm{p}}$ scatter & \multicolumn{2}{|c|}{$0.011^{a, b}$} & 1 \\
\hline$J$ & $6.992 \pm 0.023$ & $7.216 \pm 0.033$ & 5 \\
\hline$H$ & $6.726 \pm 0.039$ & $6.896 \pm 0.045$ & 5 \\
\hline$K$ & $6.627 \pm 0.033$ & $6.830 \pm 0.045$ & 5 \\
\hline$b-y$ & \multicolumn{2}{|c|}{$0.405^{a} \pm 0.006$} & 6 \\
\hline$m_{1}$ & \multicolumn{2}{|c|}{$0.214^{a} \pm 0.009$} & 6 \\
\hline$c_{1}$ & \multicolumn{2}{|c|}{$0.367^{a} \pm 0.004$} & 6 \\
\hline ST & $\mathrm{G} 2 \mathrm{~V}$ & G7V & 7 \\
\hline$M_{V}$ & $4.48 \pm 0.23$ & $4.90 \pm 0.23$ & 8 \\
\hline$T_{\text {eff }}(\mathrm{K})$ & $5989 \pm 50$ & $5713 \pm 50$ & 8 \\
\hline$\Delta T_{\mathrm{eff}}(A-B)(\mathrm{K})$ & \multicolumn{2}{|c|}{$276 \pm 14$} & 8 \\
\hline $\log g$ & $4.37 \pm 0.10$ & $4.38 \pm 0.10$ & 8 \\
\hline $\log R_{H K}^{\prime}$ & $-5.36 \pm 0.13$ & $-5.11 \pm 0.13$ & 2 \\
\hline$v \sin i\left(\mathrm{~km} \mathrm{~s}^{-1}\right)$ & $1.9 \pm 0.1$ & $2.4 \pm 0.1$ & 2 \\
\hline$v_{\mathrm{mac}}\left(\mathrm{km} \mathrm{s}^{-1}\right)$ & $4.4 \pm 0.3$ & $4.8 \pm 0.3$ & 2 \\
\hline$L_{\mathrm{X}}\left(\mathrm{erg} \mathrm{s}^{-1}\right)$ & \multicolumn{2}{|c|}{$2.1_{-1.2}^{+1.8} 10^{28 a}$} & 2 \\
\hline$[\mathrm{Fe} / \mathrm{H}]$ & $+0.29 \pm 0.10$ & $+0.20 \pm 0.10$ & 8 \\
\hline$\Delta[\mathrm{Fe} / \mathrm{H}](A-B)$ & \multicolumn{2}{|c|}{$0.091 \pm 0.009$} & 8 \\
\hline $\log N_{\mathrm{Li}}$ & 2.35 & $<1.0$ & 8 \\
\hline $\operatorname{Mass}\left(M_{\odot}\right)$ & $1.13 \pm 0.05$ & $1.06 \pm 0.05$ & 2 \\
\hline Radius $\left(R_{\odot}\right)$ & 1.05 & 0.96 & 2 \\
\hline Age (Gyr) & \multicolumn{2}{|c|}{$\sim 2$} & 2 \\
\hline
\end{tabular}

References: 1 Hipparcos (ESA 1997); 2 This paper; 3 Tycho (ESA 1997); 4 Cuypers \& Seggewiss (1999); 5 2MASS; 6 Olsen (1998); 7 Corbally \& Garrison (1980); 8 Paper I.

${ }^{a} \mathrm{~A}+\mathrm{B}$.

${ }^{b}$ See Sect. 6.2 for details.

for A and B component respectively, with an uncertainty of $0.05 M_{\odot}$ due to the errors in the parallax. In any case, the derivation of ages and masses using standard stellar models can be misleading for polluted stars (as likely HD 219542 A), that should have different composition in the radiative and convective zone (Sasselov 2002; Sandquist et al. 2002).

\subsection{Rotational profiles}

Our template spectra have resolution $(R \sim 150000)$ and $S / N$ $(\sim 200)$ high enough that the shape of the Fourier Transform of portions of spectra including suitable lines can be used to derive rotational and macroturbulent velocities.

Stellar rotational velocities have then been derived for both components of the system (A and B) using the first zeros of the Fourier Transform, as described by Gray (1992). To this purpose, we used the lines at $6252.565,6421.360$ and $6439.083 \AA$ measured on the radial velocity template spectra. Assuming a limb-darkening coefficient of 0.6 (appropriate for the stars under consideration), we obtain estimates of $1.9 \pm 0.1$ and $2.4 \pm$ $0.1 \mathrm{~km} \mathrm{~s}^{-1}$ for the values of $v \sin i$ for components A and B respectively. The value for component A agrees quite well with the value of $1.55-2.24 \mathrm{~km} \mathrm{~s}^{-1}$ obtained by Reddy et al. (2002).

The macroturbolence velocities result of $4.4 \pm 0.3$ and $4.8 \pm$ $0.3 \mathrm{~km} \mathrm{~s}^{-1}$ for HD $219542 \mathrm{~A}$ and B respectively, normal for this type of stars.

It should be noted that there is some degree of degeneracy between the values for rotational and macroturbulent velocities, that is fully removed only by using very high $S / N$ spectra. However, the macroturbolence we obtain is consistent with the spectral types of the stars, supporting the adopted values for rotational velocities.

\subsection{Activity indicators}

The level of chromospheric activity can give some clues about the age of the system and it is relevant also for the interpretation of the radial velocity behaviour of the stars (see Sect. 6). We acquired spectra of both components of HD 219542 (as well as of other $\sim 40$ stars) using the FEROS spectrograph at the $1.5 \mathrm{~m}$ telescope in La Silla in August 2002. Details of observations will be reported elsewhere. The spectra cover the range 3900-9200 A, including the region of chromospheric diagnostics $\mathrm{H}$ and $\mathrm{K} \mathrm{CaII} \mathrm{lines.} \mathrm{Figure} 1$ shows the region of the $\mathrm{K}$ line for HD 219542 A and B, compared to the solar spectrum and to the active star 36 Oph A. It appears that both stars have low chromospheric activity, with some emission possibly present in component $\mathrm{B}$ and no evidence for emission in component $\mathrm{A}$. In order to quantify the activity level, we measured on our spectra the fluxes within a $1 \AA$ band centered on the Ca II K line, and normalized them to the values of the fluxes in two narrow pseudocontinuum bands at 3909 and $3954 \AA$. This procedure was repeated for all stars observed with FEROS, as well as for solar type stars observed during the FEROS commissioning (Kaufer et al. 1999), whose spectra are available on the FEROS Consortium archives ${ }^{1}$. The resulting flux ratios $\mathrm{I}(\mathrm{K})$ were converted into $R_{H K}^{\prime}$ values by using 41 observations of 11 stars with values of $R_{H K}^{\prime}$ determined in the literature (from Baliunas et al. 1995; Henry et al. 1996, 2000). The transformation included a term dependent on $(B-V)$; this term accounts for the systematic dependences of the fluxes in the narrow reference bands on temperature and metal abundance.

\footnotetext{
${ }^{1}$ http://feros.lsw. uni-heidelberg.de/docu/ferosDB/ search.html
} 


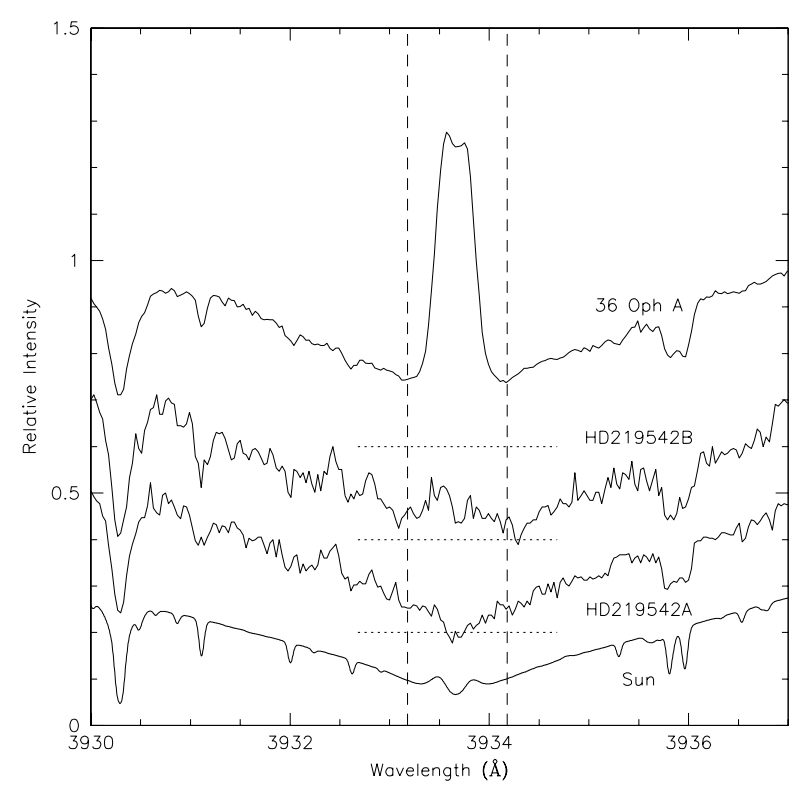

Fig. 1. The region near the core of the CaII $\mathrm{K}$ line for the Sun $\left(\log R_{H K}^{\prime}=-4.94\right)$, the components of HD 219542 and the active star 36 Oph A $\left(\log R_{H K}^{\prime}=-4.58\right.$, Henry et al. 1996). The spectra for the other stars were arbitrarily shifted in intensity with respect to the solar one. The horizontal lines represent the zero level for each spectrum. The vertical lines show the spectral region used for chromospheric activity measurement (the continuum regions are outside the plotted range).

The finally-adopted correlation is able to predict $R_{H K}^{\prime}$ values for the calibrators with an accuracy of 0.10 dex (see Fig. 2); the residual scatter is very similar to the rms scatter of $R_{H K}^{\prime}$ values for the Sun taken at different epochs (Baliunas et al. 1995), and may be attributed to the variability of the Ca II emission ${ }^{2}$. The values of $R_{H K}^{\prime}$ for HD 219542 obtained by this procedure are $-5.36 \pm 0.13$ and $-5.11 \pm 0.13$ for components A and B respectively. This low level of chromospheric activity is consistent with the results quoted by Glebocki et al. (1980). The small rotational velocities we measured are also in agreement with a low activity level for both the stars.

The ROSAT satellite detected a source (1RXS J231636.1013459) that has an offset of 13 and 18 arcsec with respect HD 219542 A and HD 219542 B respectively (Voges et al. 2000). Since the position error of the $X$-ray source is 19 arcsec, 1RXS J231636.1-013459 is very likely the counterpart of one or both the components of HD 219542 (at the high galactic latitude of this star the chance of a background object of similar flux is very small, according to Hasinger et al. 1993). The X-ray luminosity derived using the calibration of Hünsch et al. (1999) is $L_{\mathrm{X}}=2.1_{-1.2}^{+1.8} 10^{28} \mathrm{erg} \mathrm{s}^{-1}$ (A+B components). Assuming similar contribution by both components, the $\mathrm{X}$-ray luminosity results about one order of magnitude lower than Hyades stars and typical for stars with an age of about 1-2 Gyr (Randich 2000). Such X-ray luminosity is also in reasonable agreement

\footnotetext{
2 Actually, we expect that our values of $R_{H K}^{\prime}$ contain systematic errors due to stellar rotation. Lines of some of our calibrators are indeed significantly broadened by rotation; however the values of $R^{\prime}(H K)$ we derived for these stars do not differ systematically by more than 0.05 dex from the average calibration of Fig. 2.
}

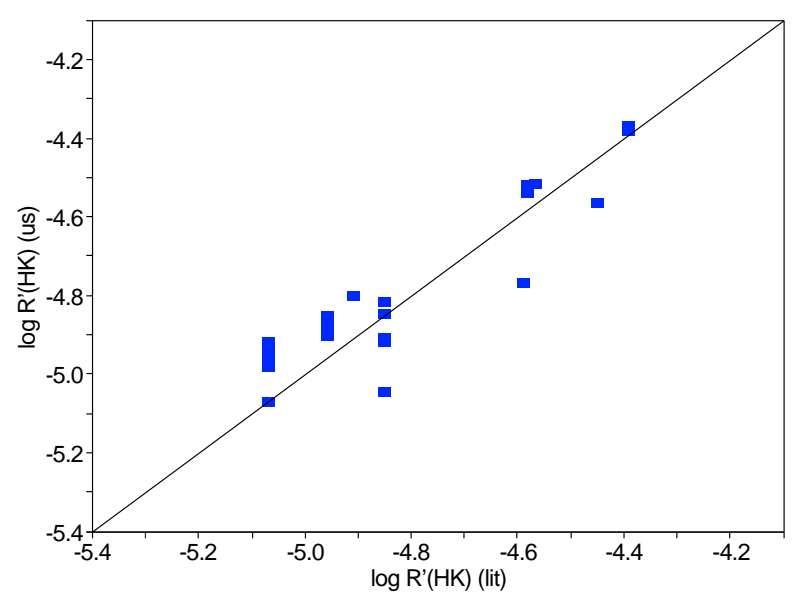

Fig. 2. The calibration of $R_{H K}^{\prime}$ index based on 11 stars with literature determinations, observed with the FEROS spectrograph during our observing run (August 2002) and instrument commissioning (Kaufer et al. 1999).

with the observed rotational velocities according to the relation by Bouvier (1990).

\subsection{The galactic orbit and the possible association with the Hyades}

Eggen (1960) in his search for moving groups in the solar neighbourhood picked up HD 219542 as a member of the Hyades moving group. While the existence itself of moving groups is debated in the literature (see Taylor 2000 and references therein), we tried to confirm or reject this possible association using updated velocities and distance for HD 219542 (see Table 1$)$. The space velocities of the binary are $(U, V, W)=$ $(-40.5,-16.5,-4.0)$. This is compatible with the space velocities of the Hyades given by Perryman et al. (1998). The galactic orbit of HD 219542, computed as in Barbieri \& Gratton (2002), is represented in Fig. 3. The orbit $\left(R_{\min }=7.70 \mathrm{kpc}\right.$; $\left.R_{\max }=9.41 \mathrm{kpc} ; Z_{\max }=0.06 \mathrm{kpc} ; e=0.10\right)$ is normal for a moderately young star.

The distance from the Sun of HD 219542 (54.6 pc) is comparable to that of the Hyades. The physical distance of HD 219542 from the center of the Hyades is about 40 pc.

Other physical indicators can be used to shed light on the possible link between the HD 219542 system and the Hyades:

- the metallicity of HD 219542 B (assumed to be unpolluted) is $[\mathrm{Fe} / \mathrm{H}]=+0.20 \pm 0.10$, compatible within errors with the cluster metallicity $([\mathrm{Fe} / \mathrm{H}]=+0.14 \text {, Perryman et al. } 1998)^{3}$; - there are no peculiar abundance patterns either in the Hyades stars or in HD 219542 B;

- the lithium content of HD $219542 \mathrm{~A}^{4}$ and especially of HD 219542 B is lower than that of Hyades stars of similar temperature (Fig. 4) and rather similar to that of stars in

\footnotetext{
${ }^{3}$ Note that the $[\mathrm{Fe} / \mathrm{H}]$ values given in Paper I may be slightly overestimated, due to the choice of a very low value for the microturbulent velocity.

4 The measurement of lithium of HD 219542 A of Paper I was confirmed by the independent analysis by Reddy et al. (2002).
} 


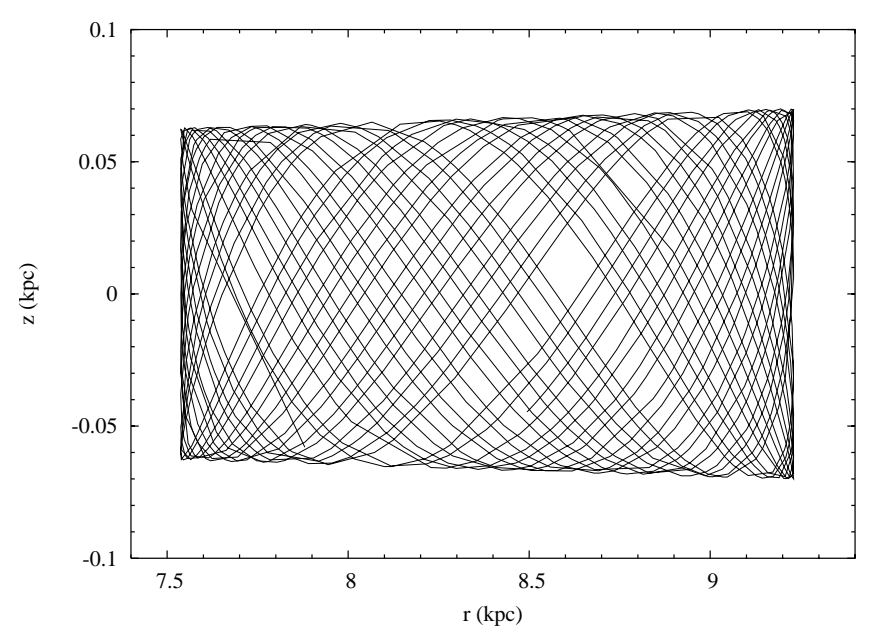

Fig. 3. Galactic orbit of HD 219542 in the meridional plane.

older open clusters like NGC 752 and M 67 (Paper I). This seems not compatible with the Hyades association, since Hyades show a very low scatter in lithium abundance at a given temperature;

- isochrone fitting does not have the accuracy for distentangling ages differences of $\sim 0.5$ Gyr for unevolved objects like the two components of HD 219542;

- the rotational velocities of the components of HD 219542 are at the low end of those of Hyades stars of similar spectral type. Note that a pole-on orientation for both components is unlikely, since for binaries with separation larger than $40 \mathrm{AU}$ the rotation axes do not appear to be aligned (Hale 1994);

- the chromospheric activity as measured on our FEROS spectra is much lower than that of Hyades stars of similar colour;

- the X-ray luminosity derived in Sect. 2.3 is about one order of magnitude lower than that of Hyades stars of similar temperature;

- none of the 24 Hyades G dwarfs studied by Paulson et al. (2002) has a radial velocity jitter as low as HD 219542 A (see Sect. 6.4).

In summary, we conclude that while the kinematic data suggests the HD 219542 may be part of the Hyades moving group, the other stellar properties (lithium, rotation, chromospheric activity, X-ray emission, radial velocity jitter) seems to indicate a much older age (at least 1-2 Gyr) for the system. In particular, isochrone fitting and X-ray emission point to an age of $\sim 1-2$ Gyr, while the very low chromospheric activity suggests an even older age.

\section{Orbital properties of the binary system}

\subsection{Astrometric data}

The projected separation of 5.279 arcsec at the distance measured by Hipparcos (54.6 pc) corresponds to $288 \mathrm{AU}$. Available data can be used to costrain the orbit of the binary. The first astrometric measurement of this pair dates back to 1830 (Aitken 1932). The literature astrometric measurements are listed in

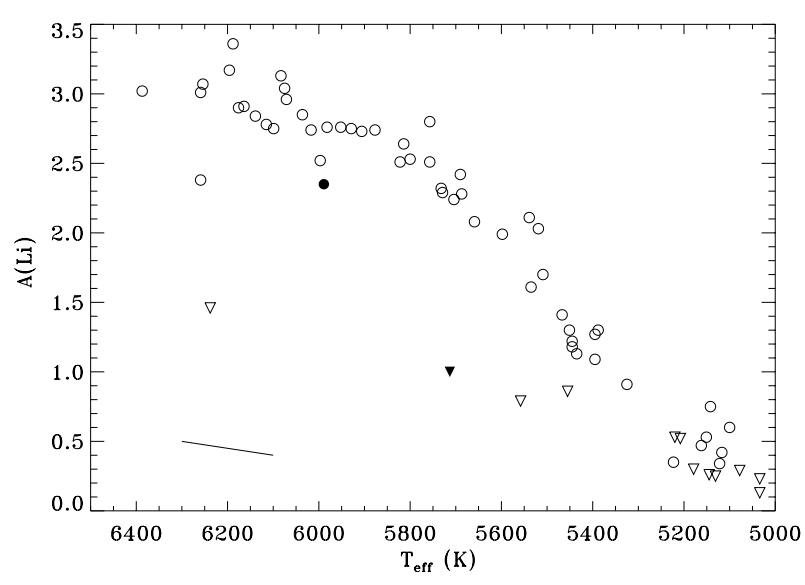

Fig. 4. Lithium determinations for Hyades stars from Thorburn et al. (1993) (empty circles for abundance measurements and empty triangles for upper limits) and for the components of HD 219542 (HD 219542 A: filled circle; HD 219542 B: filled triangle). Only an upper limit was derived for HD 219542 B. The line at the lower left corner represents variations of derived lithium content as a function of the adopted effective temperature. Even assuming large differences in the temperature scales used by Thorburn et al. (1993) and Paper I of $\sim 150-200 \mathrm{~K}$, it is not possible to obtain a lithium content for HD 219542 similar to that of the stars of Hyades of similar temperature.

Table 2. Relative position of the components of HD 219542.

\begin{tabular}{ccccccc}
\hline \hline Epoch & $\rho$ & Error & $\theta$ & Error & $\theta_{2000.0}$ & Ref. \\
\hline 1830.51 & 4.56 & & 26.7 & & 26.5 & 1 \\
1890.64 & 4.75 & & 30.6 & & 30.5 & 1 \\
1909.05 & 4.96 & & 29.9 & & 29.8 & 1 \\
1917.32 & 4.96 & & 30.3 & & 30.2 & 1 \\
1924.40 & 4.97 & & 29.2 & & 29.1 & 1 \\
1953.545 & 5.103 & 0.013 & 30.94 & 0.16 & 30.89 & 2 \\
1960.699 & 5.11 & 0.02 & 30.8 & 0.2 & 30.8 & 3 \\
1976.784 & 5.02 & & 31.3 & & 31.3 & 4 \\
1991.25 & 5.279 & 0.004 & 31.53 & & 31.52 & 5 \\
1991.797 & 5.280 & 0.002 & 31.47 & 0.02 & 31.46 & 6 \\
1994.747 & 5.29 & 0.20 & 32.05 & 0.78 & 32.05 & 7 \\
\hline
\end{tabular}

References: 1 Aitken (1932); 2 van Albada-van Dien (1978); 3 van Albada-van Dien (1983); 4 Holden (1977); 5 Hipparcos (ESA 1997) 6 Cuypers \& Seggewiss (1999); 7 Popovic \& Pavlovic (1997).

Table 2. Position angles were precessed to 2000.0 using Eq. (5) of Heintz (1978).

Clear trends both in separation and in position angle can be noticed (Fig. 5). No changes in separation and position angle were instead detected by Hipparcos because of its limited time baseline. A linear fitting to the available data ${ }^{5}$ gives $\mathrm{d} \rho / \mathrm{d} t=$ $0.0048 \pm 0.0003 \mathrm{arcsec} / \mathrm{yr}$ and $\mathrm{d} \theta / \mathrm{d} t=0.018 \pm 0.003 \mathrm{deg} / \mathrm{yr}$.

\footnotetext{
5 The measurements were weighted according to their errors, when available, and assigning errors of $0.2 \operatorname{arcsec}$ and $2 \operatorname{deg}$ for $\rho$ and $\theta$ measurements respectively for those stars without errors bars.
} 


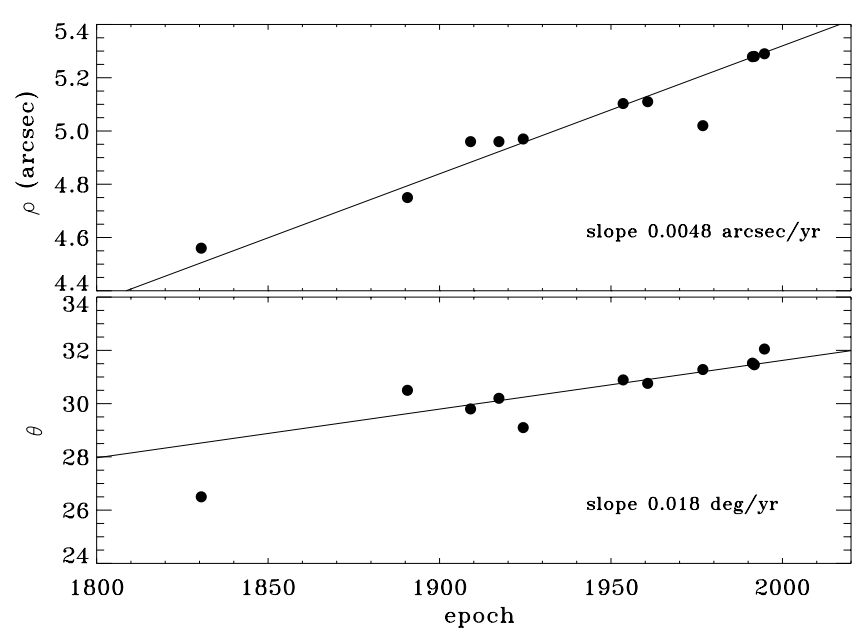

Fig. 5. Astrometric measurements of HD 219542. Overplotted is the best linear fit to the data.

Table 3. Relative position and velocities of the components of HD 219542 in the cartesian plane.

\begin{tabular}{ccc}
\hline \hline Parameter & Value & Error \\
\hline$x(\mathrm{AU})$ & 246 & 26 \\
$y(\mathrm{AU})$ & 151 & 16 \\
$z_{\min }(\mathrm{AU})$ & -1260 & 280 \\
$z_{\max }(\mathrm{AU})$ & 1260 & 280 \\
$v_{x}\left(\mathrm{~m} \mathrm{~s}^{-1}\right)$ & 840 & 140 \\
$v_{y}\left(\mathrm{~m} \mathrm{~s}^{-1}\right)$ & 1020 & 110 \\
$v_{z}\left(\mathrm{~m} \mathrm{~s}^{-1}\right)$ & -1150 & 25 \\
\hline
\end{tabular}

\subsection{Radial velocity difference}

A further clue to the orbital properties of the system comes from the radial velocity differences between the components, that results $-1.15 \pm 0.02 \mathrm{~km} \mathrm{~s}^{-1}$ (see Sect. 4.3). Therefore HD $219542 \mathrm{~B}$ is moving toward us with respect to HD $219542 \mathrm{~A}$.

\subsection{Clues on the orbital parameters}

The availability of $\rho, \theta$, their time derivatives, the velocity difference and the parallax means that we know the relative position of the stars in the plane of the sky $(x, y)$ and all the velocity components $v_{x}, v_{y}, v_{z}$ (Table 3 ). The position $z$ along the line of sight is instead unknow, so that it is not possible to derive the orbital parameters of the visual binary.

However, it is possible to constrain the value of $z$ assuming that the two components are bound and derive the orbital parameters as a function of $z$. To this aim, we follow the procedure by Hauser \& Marcy (1999). The allowed separation along the line of sight results $-1260<z<1260$ AU. The orbital parameters as a function of $z$ are shown in Fig. 6 .

It appears that low eccentricity orbits with periastron of a few hundreds AU, as well as very eccentric orbits with periastron as small as $10 \mathrm{AU}$, are compatible with the available data. Radial velocity variations due to the binary orbit are
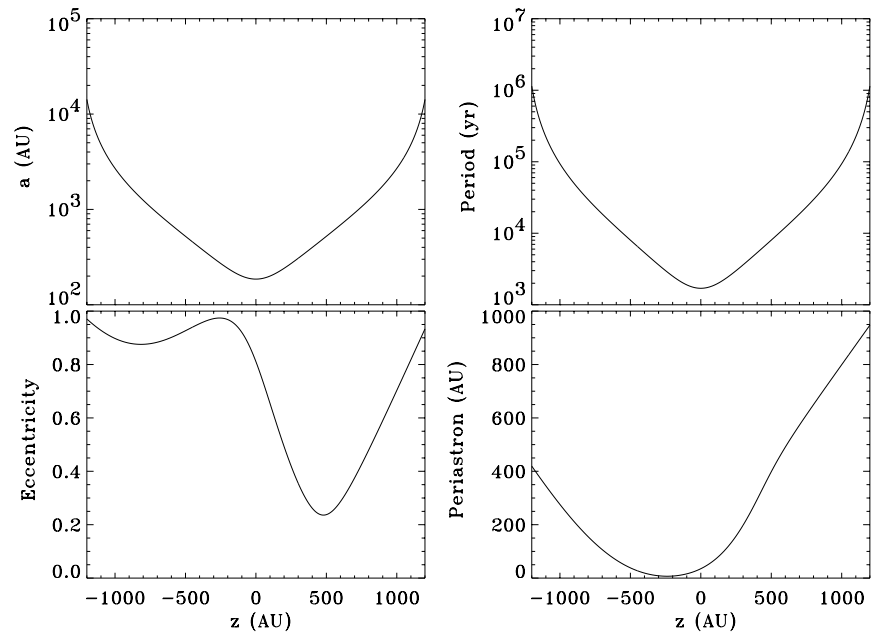

Fig. 6. Possible values of semimajor axis, period, eccentricity and periastron distance of the orbit of HD 219542 as a function of the (unknown) separation along the line of sight.

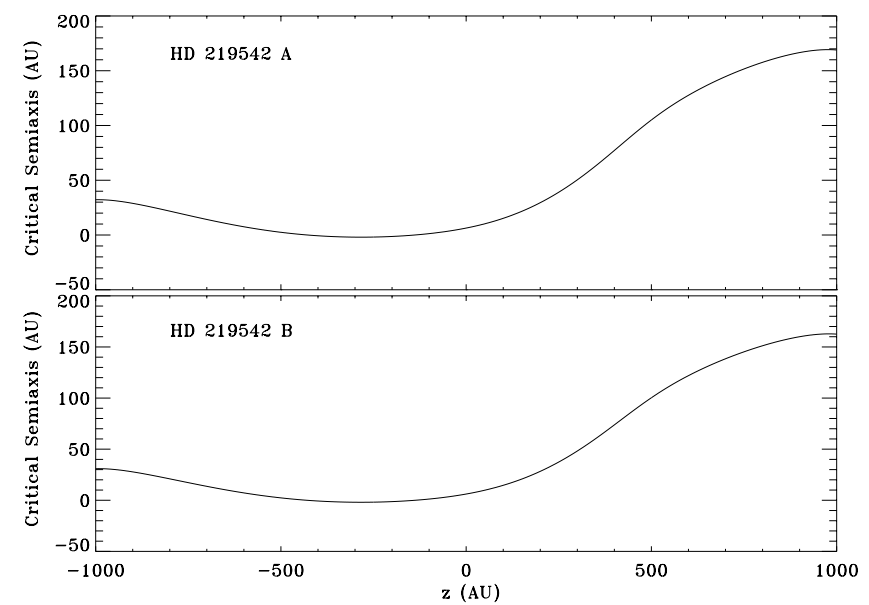

Fig. 7. Critical semiaxis for dynamical stability of planets orbiting HD 219542 A (upper panel) and HD 219542 B (lower panel) as a function of the current separation along the line of sight.

below $1 \mathrm{~m} \mathrm{~s}^{-1} \mathrm{yr}^{-1}$ over the whole range of $z$, undetectable from our data.

The critical semiaxis for dynamical stability of planets for the family of orbits described above can be calculated following Holman \& Weigert (1999). As expected, for the very eccentric orbits occurring for $-500<z<0$ AU the zone allowed for stable planetary orbits is very small, while for the low eccentricy orbits occurring if $z \sim 500$ AU dynamical stability up to $\sim 100 \mathrm{AU}$ is ensured (Fig. 7).

\section{Radial velocities}

\subsection{Observations and data analysis}

The components of HD 219542 were observed as part of our ongoing planet search program using SARG at TNG. The goals of the project are the study of the frequency of planets around stars in wide binaries with a typical separation 200-300 AU, looking for the dynamical effects due to the stellar companion, and the search for chemical differences between the 
components (see Paper I). A recent report is presented by Gratton et al. (2002). The survey as well as the observational and data analysis procedure will be described in detail in a forthcoming paper (Desidera et al., in preparation). Here we briefly summarize the most relevant points.

The observations used for the radial velocity determinations were acquired with the SARG spectrograph using the Yellow Grism, that covers the spectral range 4600-7900 $\AA$ without gaps, and the 0.25 arcsec slit. The resulting resolution is $R=150000$ ( 2 pixels sampling). The iodine cell was inserted into the optical path, superimposing a dense forest of absorption lines used as reference spectrum for the radial velocity determination. The spectral ranges $4600-5000 \AA$ and 6200-7900 $\AA$ are free from the contamination of iodine lines and can be used to monitor the changes of the line profile and equivalent width (see Sects. 6.1-6.3). Only the blue part of the spectra is used in this paper. The insertion of a suitable filter avoids any grism second order contamination in the red extreme of the spectra. Exposure times were fixed at $15 \mathrm{~min}$, to reduce the errors in barycentric correction caused by the lack of knowledge of the exact flux mid time of the exposure. During the observations, the slit was usually oriented perpendicularly to the separation of the components to minimize the contamination of the spectra by the companion. The rather large separation of the components of HD 219542 (5.3 arcsec) makes the contamination issue not important for the case presented here.

The data reduction was performed using $\mathrm{IRAF}^{6}$.

The radial velocity analysis was performed using the AUSTRAL code developed by Endl et al. (2000). Each star + iodine spectrum was modeled using a pure iodine template of the SARG cell obtained with the Kitt Peak FTS (Desidera 2000) and a high quality pure stellar template (i.e. without the iodine cell in the path) obtained with SARG, properly deconvolved for the instrument profile. In the modeling of the star + iodine spectrum, the best Doppler shift of the stellar spectrum relative to its template is found and the instrument profile was reconstructed. Since the instrument profile changes along a spectral order, the spectrum was divided in chunks approximately 100 pixels long $(\sim 2 \AA)$, each one modeled independently. About 400 chunks were used in the final analysis. Internal errors of velocities are calculated as the errors of the mean of the chunks. We find that averaging the velocities obtained with several different chunk lengths (in the range 90-120 pixels for solar type stars) improves the quality of the results. Changing the chunk length means to slightly reduce the impact of noise due to the division of the spectrum in discrete pieces.

The parameters for the instrument profile modeling were instead kept fixed on values fine-tuned using fast rotating featureless stars, the constant radial velocity star $\tau$ Ceti and the planet-bearing stars $51 \mathrm{Peg}$ and $\rho \mathrm{CrB}$. A long term precision of about $3 \mathrm{~m} \mathrm{~s}^{-1}$ was achieved for these stars. For the much fainter $(V=8-9)$ stars of the binary sample the errors are typically about $5 \mathrm{~m} \mathrm{~s}^{-1}$, due to the lower $S / N$ of the spectra.

\footnotetext{
${ }^{6}$ IRAF is distributed by the National Optical Observatory, which is operated by the Association of Universities for Research in Astronomy, Inc., under contract with the National Science Fundation.
}

Table 4. Differential radial velocities of HD 219542 A.

\begin{tabular}{ccc}
\hline \hline JD-2450000 & $R V$ & error \\
\hline 1825.52 & -7.2 & 5.8 \\
1826.59 & -6.0 & 8.6 \\
2070.70 & -1.9 & 3.8 \\
2071.70 & 1.9 & 3.9 \\
2072.68 & 1.0 & 4.5 \\
2113.69 & -2.4 & 4.1 \\
2115.67 & -3.8 & 4.5 \\
2115.71 & 4.0 & 5.1 \\
2116.56 & 5.8 & 8.2 \\
2116.70 & -1.4 & 5.6 \\
2117.68 & 5.2 & 3.9 \\
2120.71 & 1.1 & 5.0 \\
2145.65 & -1.4 & 4.0 \\
2190.53 & 4.0 & 4.5 \\
2216.46 & -5.5 & 7.7 \\
2245.42 & -3.1 & 4.4 \\
2424.70 & 3.7 & 4.6 \\
2445.70 & 11.6 & 4.5 \\
2472.70 & 7.9 & 6.0 \\
2504.67 & -7.2 & 11.0 \\
2538.50 & -7.5 & 5.9 \\
2570.40 & -2.1 & 4.9 \\
2585.46 & 12.9 & 7.3 \\
2597.36 & -2.3 & 5.2 \\
2605.35 & -7.3 & 5.1 \\
\hline & &
\end{tabular}

For the components of HD 219542, the typical $S / N$ we achieved is $80-100$. The stellar templates (used also in Paper I for the abundance analysis) have instead $S / N$ above 200 .

\subsection{Differential radial velocities}

More than 20 radial velocities measurements of the components of HD 219542 were obtained, spanning the period October 2000-November 2002 (Tables 4, 5). On a few nights, we obtained two consecutive spectra for the targets. In these cases, we computed a weighted average of the velocities.

The time series of velocities of HD $219542 \mathrm{~A}$ is shown in Fig. 8. The dispersion of velocities is $5.8 \mathrm{~m} \mathrm{~s}^{-1}$, only slightly larger than the mean internal errors $\left(5.6 \mathrm{~m} \mathrm{~s}^{-1}\right)$ determined by the chunk-to-chunk internal scatter in the velocities ${ }^{7}$. Statistical analysis (see Sect. 5) does not reveal significant periodicities. Long term trends are also not apparent from the data. Therefore we can rule out both short-period Jupiter-mass planets as well as massive companions in external orbits. The upper limits on the giant planets around HD 219542 A still compatible with the data are discussed in Sect. 7.

The velocities of HD 219542 B show instead a dispersion $\left(9.2 \mathrm{~m} \mathrm{~s}^{-1}\right)$ larger than the measurement errors $\left(5.1 \mathrm{~m} \mathrm{~s}^{-1}\right)$.

\footnotetext{
${ }^{7}$ Comparison between pairs of observations obtained consecutively in both components suggest that the errors given by this procedure may be overestimated by about $25 \%$, possibly because individual chunks consistently deviates by similar amounts in spectra acquired on the same night.
} 
Table 5. Differential radial velocities of HD 219542 B.

\begin{tabular}{|c|c|c|}
\hline JD-2 450000 & $\overline{R V}$ & error \\
\hline 1825.51 & -4.4 & 7.5 \\
\hline 1826.49 & -11.9 & 5.9 \\
\hline 2070.71 & 1.5 & 4.1 \\
\hline 2071.71 & 6.6 & 4.0 \\
\hline 2072.69 & 8.9 & 5.9 \\
\hline 2113.71 & -0.2 & 4.6 \\
\hline 2115.69 & 2.4 & 5.7 \\
\hline 2115.71 & 8.6 & 5.2 \\
\hline 2116.68 & -6.6 & 5.8 \\
\hline 2117.70 & -3.8 & 7.8 \\
\hline 2117.71 & 2.5 & 6.8 \\
\hline 2120.72 & 2.4 & 5.0 \\
\hline 2145.66 & -2.2 & 4.6 \\
\hline 2190.54 & 3.1 & 3.8 \\
\hline 2216.47 & 9.4 & 7.2 \\
\hline 2245.43 & -8.4 & 4.3 \\
\hline 2424.71 & 11.4 & 5.2 \\
\hline 2445.71 & 13.2 & 4.5 \\
\hline 2472.70 & -8.6 & 5.2 \\
\hline 2538.51 & 23.4 & 7.9 \\
\hline 2570.41 & -7.8 & 5.2 \\
\hline 2570.42 & -0.7 & 4.9 \\
\hline 2585.44 & -16.4 & 7.3 \\
\hline 2585.45 & -18.4 & 6.7 \\
\hline 2597.33 & -1.9 & 4.4 \\
\hline 2597.34 & 5.3 & 4.8 \\
\hline 2605.36 & -4.8 & 4.8 \\
\hline 2605.37 & -2.8 & 5.3 \\
\hline
\end{tabular}

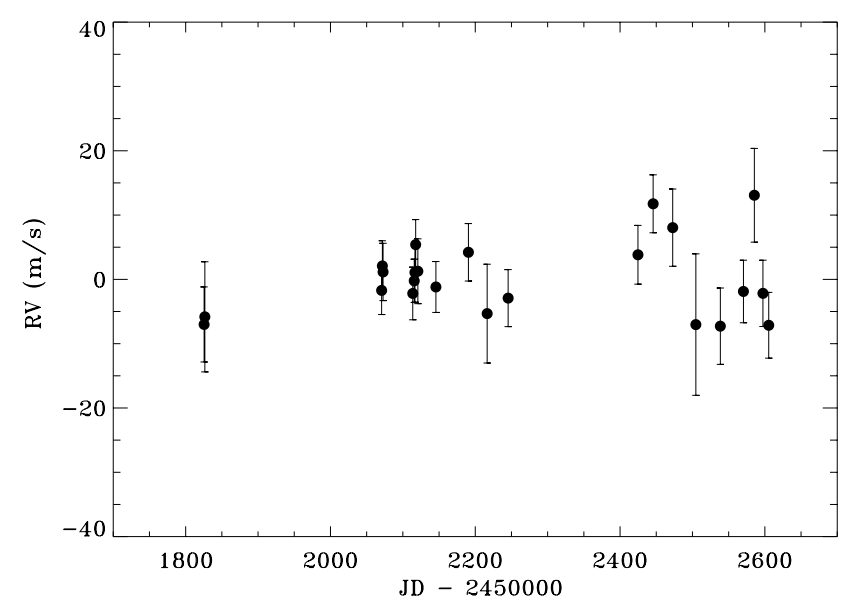

Fig. 8. Differential radial velocities of HD 219542 A. The dispersion of the velocities is $5.8 \mathrm{~m} \mathrm{~s}^{-1}$, compatible with measurement errors.

Since the two components were usually observed consecutively, we can exclude the occurrence of systematic errors due to uncorrected instrumental shifts or barycentric correction as possible causes of the observed variations.

We performed a period search within the $R V$-results in the range of 2 to 1000 days, using the well-known LombScargle periodogram (Lomb 1976; Scargle 1982). We found a strong peak at a period of 112 days. Its significance is
Table 6. Possible orbital solution for the velocity of HD 219542 B.

\begin{tabular}{lc}
\hline \hline Parameter & Value \\
\hline Period $(\mathrm{d})$ & $112.1 \pm 1.2$ \\
Semi-amplitude $\left(\mathrm{m} \mathrm{s}^{-1}\right)$ & $13.0 \pm 3.0$ \\
Eccentricity & $0.32 \pm 0.15$ \\
Longitude of periastron $(\mathrm{deg})$ & $51 \pm 33$ \\
Periastron passage $(\mathrm{JD}-2450000)$ & $1770.6 \pm 8.0$ \\
& \\
$a(\mathrm{AU})$ & 0.46 \\
$m \sin i$ & 0.30 \\
\hline
\end{tabular}

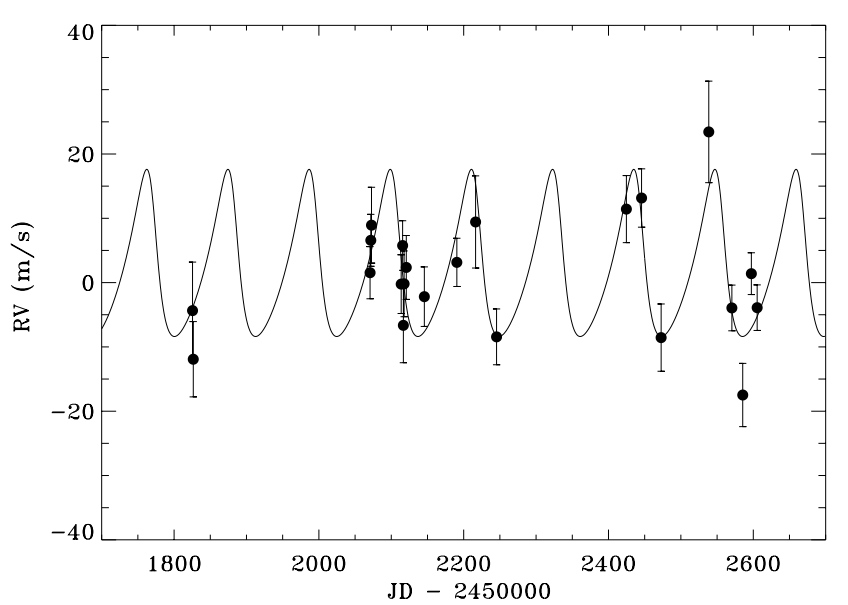

Fig. 9. Differential radial velocities of HD 219542 B. Overplotted is the best Keplerian solution.

discussed in Sect. 5. The best-fit Keplerian orbital solution ${ }^{8}$ to the RV-data of HD 219542 B yields a period $P=112.1$ days, semiamplitude $K=13.0 \mathrm{~m} \mathrm{~s}^{-1}$ and a moderate eccentricity $e \sim 0.32$ (Table 6 ). The rms of residuals from the Keplerian fit is $5.1 \mathrm{~m} \mathrm{~s}^{-1}\left(5.9 \mathrm{~m} \mathrm{~s}^{-1}\right.$ taking into account the loss of degrees of freedom introduced by the Keplerian fitting). Figure 9 shows the radial velocities of HD 219542 B with overplotted the orbital solution; Fig. 10 shows the residuals from the Keplerian fit, and Fig. 11 the velocities of HD 219542 B phased to best fitting period. Fischer et al. (2001) noted that for noisy data (and in the case of undetected further planets in the system) the orbital fitting produces spurious eccentricity enhancements. For this reason, the derived eccentricity should be considered with some caution.

Among the planets found so far, 3 have comparable velocity amplitude (namely HD 16141b, Marcy et al. 2000; 47 UMa c, Fischer et al. 2002; 55 Cnc c, Marcy et al. 2002). Therefore a careful evaluation of the data is required to discuss the significance of the result (Sect. 5) and the possible activity origin of the observed variations (Sect. 6). As we will see, the results of these tests are rather inconclusive, so that the existence of a planet around HD 219542 B still needs confirmation ${ }^{9}$.

\footnotetext{
8 The fitting was performed using the program Orbitsolver (R.E.M. Griffin, priv. comm.) that adjusts all the elements of the orbit simultaneously in a least-squares solution (Griffin \& Cornell 1998).

${ }^{9}$ We cannot then at present confirm at a high level of confidence the announcement of a planet that some of us (the Italian part of the group) gave as a press release in November 2002.
} 


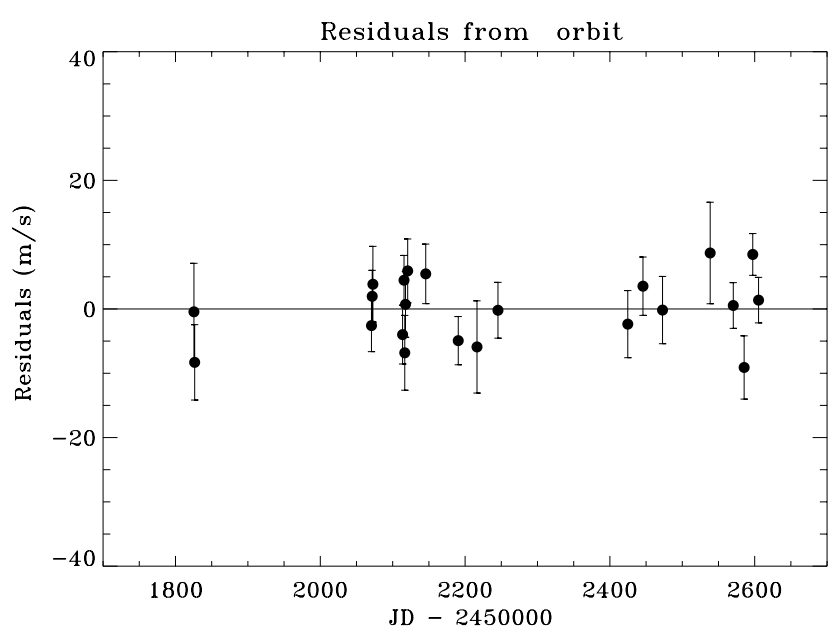

Fig. 10. Residuals of the velocities of HD 219542 B from the calculated orbit.

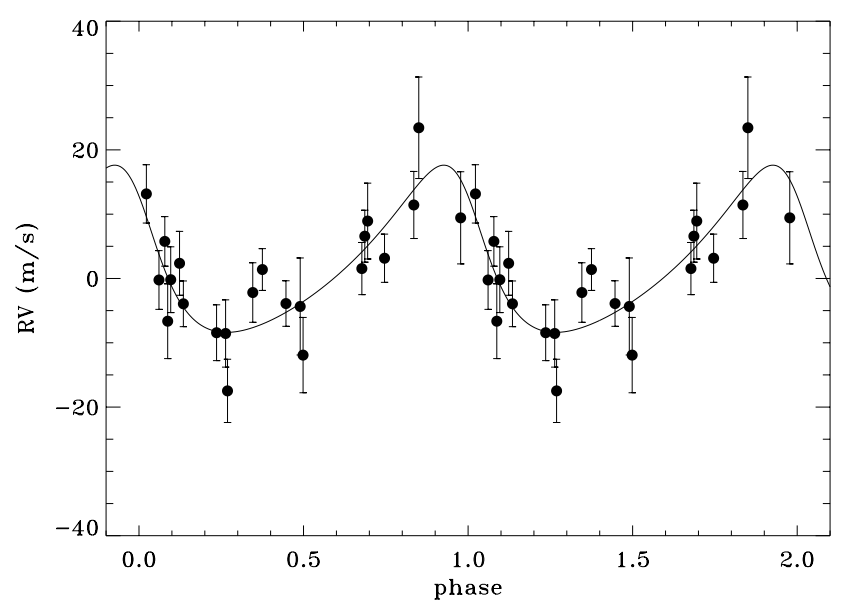

Fig. 11. The velocities of HD 219542 B phased to the 112.1 days period of the best orbital solution.

\subsection{Absolute radial velocities}

The iodine cell technique provides velocities relative to an arbitrary zero point, different for each star. Cross-correlation with a template of a star with known absolute radial velocity allows to determine the absolute velocity values for both stars.

We used as template $51 \mathrm{Peg}$, that has an absolute velocity of $-33.23 \mathrm{~km} \mathrm{~s}^{-1}$ (Nidever et al. 2002). The velocities result of $V_{\mathrm{A}}=-11.1 \mathrm{~km} \mathrm{~s}^{-1}$ and $V_{\mathrm{B}}=-10.0 \mathrm{~km} \mathrm{~s}^{-1}$. Corrections for gravitational redshift and convective blueshift were not included, because 51 Peg has a colour intermediate between those of HD $219542 \mathrm{~A}$ and B. It was also not necessary to include a correction for the orbital motion due the planet around $51 \mathrm{Peg}$, because the template of $51 \mathrm{Peg}$ was acquired by chance with the star close to its center of mass velocity. The errors of our velocities are likely within $0.2 \mathrm{~km} \mathrm{~s}^{-1}$. The only literature determination of radial velocity is $-16.5 \mathrm{~km} \mathrm{~s}^{-1}$ for HD 219542 A (Wilson 1953). The discrepancy is likely explained by the low accuracy of the old data.

For the analysis of the possible binary orbit (Sect. 3) it is important to have a precise measurement of the difference of radial velocities between the components. Such a difference was measured using the same stellar template for both stars. Considering the templates of HD 219542 A and HD 219542 B, the radial velocity difference results $V_{\mathrm{A}}-V_{\mathrm{B}}=-1200 \pm$ $10 \mathrm{~m} \mathrm{~s}^{-1}$. Such quantity must be corrected for the difference of the gravitational redshift and convective blueshift between the stars to give the real radial velocity difference. We estimate this effect to be about $50 \mathrm{~m} \mathrm{~s}^{-1}$, using the relation by Nidever et al. (2002). Therefore our best estimate for the radial velocity difference between the components is $V_{\mathrm{A}}-V_{\mathrm{B}}=-1150 \pm$ $25 \mathrm{~m} \mathrm{~s}^{-1}$ (including further $\sim 20 \mathrm{~m} \mathrm{~s}^{-1}$ of uncertainty in the gravitational and convective corrections).

\section{Test of significance of the proposed planet orbit}

We considered two different tests to evaluate the significance of the solution. The first one answers to the question: "What is the probability that we would extract a solution corresponding to a Keplerian motion induced by a planet better than the solution found from the real data from a similar analysis of a random sequence of radial velocities sampled at the same epochs and with the same noise as the data?"

The following procedure was used to answer this question. We employed an orbit finder program that scans through the parameter range for period, amplitude, phase angle, eccentricity and periastron angles and determines the quality of the fit for each test-orbit. We considered two different best fit estimators, minimizing either the rms scatter of data or the $\chi^{2}$ of the distribution, using the internal errors as estimate of the real errors. While $\chi^{2}$ might be expected to be a better fit estimator, we note that on one side the distribution of individual internal errors is quite uniform, and on the other internal errors may neglect some source of scatter present in the data (e.g. activityinduced jitter, barycentric correction, etc.). Furthermore, we have some indications that internal errors are overestimated by $\sim 25 \%$ (Sect. 4.2). We define as acceptable those solutions whose eccentricity is not larger than 0.8 , and there is no phase interval longer than 0.25 without any data.

We verified that our orbit finder program delivers similar solutions to the OrbitSolver program using both estimators. The solutions found by our program have in both cases period of $111.3 \mathrm{~d}$, amplitude of $12.7 \mathrm{~m} \mathrm{~s}^{-1}$, and eccentricity 0.4 . The rms of residuals is of $5.8 \mathrm{~m} \mathrm{~s}^{-1}$ and the $\chi^{2}$ is $104.7 \mathrm{~m} \mathrm{~s}^{-1}$. In both cases they are very close to the optimized value.

We employ a bootstrap randomization scheme in order to estimate the significance of the 112 days period. In this bootstrap method the radial velocities and errors of individual nights are randomly re-distributed along the times of observations.

We ran the program on the generated data sets and we found that only in 2 cases out of 1000 , random extractions yield solutions with an rms smaller than or equal to that given by the real observations. When using $\chi^{2}$, the solutions better than the observed case are $1.2 \%$.

Second, we tested the probability to have by chance a power in the periodogram similar to that of the observed data, following the bootstrap approach decribed above and computing the power spectrum for each new dataset is generated (Kürster et al. 1997; Murdoch et al. 1993). For each of the two components 


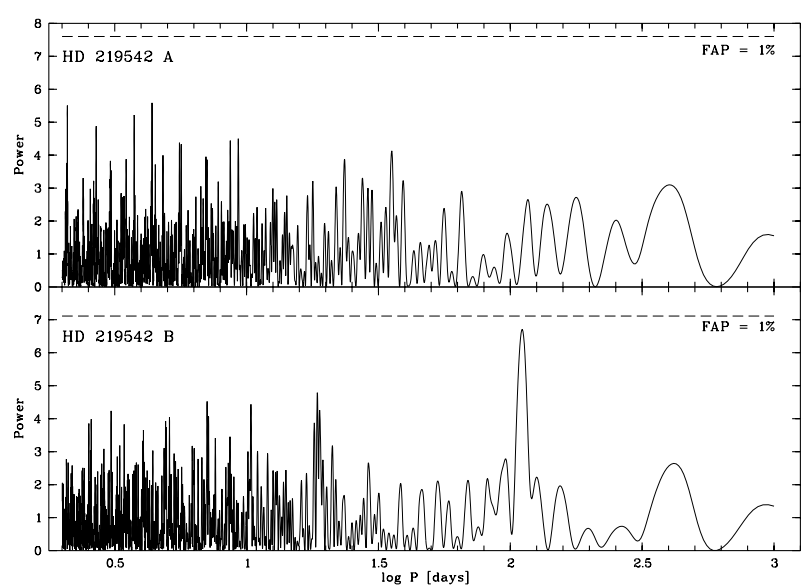

Fig. 12. Lomb-Scargle periodogram results for the $R V$ data of component A (upper panel) and component B (lower panel). The falsealarm-probability (FAP) levels of $1 \%$ are indicated as horizontal dashed lines (see text for details). While there is no signal present for HD 219542 A, we find a strong peak at $P=111$ days for HD 219542 B. However, even this signal has a FAP of $3.7 \%$ and is thus not highly significant.

we performed 10000 bootstrap runs. The results are displayed in Fig. 12. We found a false-alarm-probability (FAP) of $3.7 \%$. We note that this second approach is more general, since it assumes that the signal is given by sine-functions, independently of its physical origin. From this more prudent estimate, we conclude that there is a $3 \%$ probability that our solution may be due to chance.

The same tests performed for component $\mathrm{A}$ give a rather high probability (more than $50 \%$ ) that a better result can be obtained from a random set of radial velocities.

Finally, it should also be remembered that a basic assumption throughout this procedure is that there is no correlation between noise on consecutive (or close) nights. However, some correlation is expected if noise is dominated by activity. Hence this test cannot exclude that the observed velocity variations are due to activity rather than to Keplerian motion.

\section{Activity or Planet?}

The low amplitude of the velocity variations of HD 219542 B may be in principle due to orbital motion (i.e. a planet) or to activity related phenomena. We note that a period of 112 days is too long for a rotational modulation (the upper limit to the rotational period from the observed $v \sin i$ value is $\sim 21$ days) and too short for the long term activity cycle typical for solar type stars. However, it is still possible that the observed variations are due to some transient short period modulations, not apparent as significant in the statistical analysis because of the lack of coherence over our $\sim 800$ days of observations ${ }^{10}$. In this case, however, we would have picked up a phase of low activity during our observations in July 2001, when we obtained

\footnotetext{
10 The second peak in Fig. 12 is at 18.5 days (a reasonable rotational period for the star). While the season 2002 data can be fitted fairly well by a sinusoidal variation with this period, the 2001 data cannot.
}

5 observations over 8 nights, showing a dispersion of $4.5 \mathrm{~m} \mathrm{~s}^{-1}$ (nightly averages).

We performed a few tests to check this possibility: we studied the characteristics of the components of HD 219542 to infer the expected activity-induced radial velocity jitter, we checked the Hipparcos photometry for variability, and we measured line bisector and equivalent width variability on SARG spectra.

\subsection{Bisector analysis}

Given the small amplitude of the observed variations in the radial velocity of HD $219542 \mathrm{~B}$, it is well possible that such variations are not real, but rather are due to the effects of line asymmetries. Such variations could be in principle related to activity: e.g. in the case of HD 166435, Queloz et al. (2001) found that radial velocity variations with semiamplitude as large as $K=83 \mathrm{~m} \mathrm{~s}^{-1}$ can be attributed to correlated variations of the line bisectors of $\sim 200 \mathrm{~m} \mathrm{~s}^{-1}$ (peak-to-peak) with the rotational period and attributable to the effects of star spots. Unfortunately, such an analysis is much more difficult in the present case, because the variations in the bisectors required to explain the radial velocity variations are very small $\left(\leq 10 \mathrm{~m} \mathrm{~s}^{-1}\right)$. While we did not expect that such an accuracy can be achieved using our data for both components of HD 219542, we however performed an analysis of the variations of line bisectors, using those orders shortward of $5000 \AA$, where there are not lines due to iodine.

The technique we used is similar to that devised by Queloz et al. (2001), that is an analysis of asymmetries in the Cross Correlation Function $(\mathrm{CCF})$. The $\mathrm{CCF}$ is obtained by cross correlating the observed spectra with a suitable mask, that is a function that is 1 within very narrow regions (ideally $\delta$ of Dirac) centered at the wavelengths of selected spectral lines, and 0 elsewhere. This CCF represents an average profile for the lines in the lists. To this purpose we selected about 80 clean spectral lines in this spectral region, with equivalent widths in the range $10-70 \mathrm{~mA}$. The left panel of Fig. 13 displays a typical CCF obtained by this technique. Typical Full Width Half Maximum $(F W H M)$ of our CCF's are $0.10 \AA$ : this broadening is essentially due to macroturbulence, since the instrumental profile has a typical $F W H M$ of $0.032 \AA$. In analogy with Queloz et al. (2001), we quantified line asymmetry span as the difference $b$ between the average bisector values in two regions of the CCF's (from 0.96 to 0.89 , and from 0.86 to 0.72 of the line profile, see Fig. 13).

Our analysis is certainly not optimal: in fact, asymmetries in our CCFs may arise for reasons different from real asymmetries in the spectral lines (and other than photon noise). Possible causes include blends with nearby lines, errors in the wavelength calibration and inhomogeneities in the slit illumination. The first effect has not a large impact here, because it would show up as a constant pattern, and we are rather interested in bisector variations. Also, we selected lines very carefully, and the average line profiles is very close to the continuum at the base of the line. We also note that at least in the case of 51 Peg (for which we performed a similar analysis using our own spectra, see below), the run of the bisector 




Fig. 13. Left panel: Cross Correlation Function for a spectrum of HD 219542 B; right panel: superposition of the the mean line bisectors from the spectra of 51 Peg (dotted line), HD 219542 A (dashed line), and HD 219542 B (solid line). T and B regions are those used in the computation of the bisector span.

with depth is similar to those published in the literature (Gray 1997) in the regions of overlap. The runs of the bisectors with depth are very similar for $51 \mathrm{Peg}$ and the two components of HD 219542, with a trend for the span to slightly larger in the warmer star (HD 219524 A) and slightly smaller in the cooler one (HD 219542 B), with 51 Peg (which has intermediate temperature) showing an intermediate behaviour. This agrees with expectations (Gray 1992). The other effects are of major concern, and likely are more important in our analysis than in that by Queloz et al. (2001) because slit illumination is not scrambled by fibers, and because our wavelength calibration is not as accurate. On the other side, it should be also noted that our spectra have a resolution that is about three times larger. This would result in a gain af about one order of magnitude of the sensitivity of the CCF to real changes in the bisectors. We also note that all these effects do not affect our radial velocities, because they are virtually eliminated in our calibration procedure based on the iodine cell lines; however they may dominate asymmetries in the cross correlation peaks, hiding the effects of activity-related variations of the bisectors.

In order to quantify the internal errors, we analyzed with the same procedure a series of 23 spectra of $51 \mathrm{Peg}$ (these spectra have $S / N$ ratios about 2.5 times larger than those of HD 219542) taken over two years with SARG at TNG. Spectrum-to-spectrum variations of the bisector span $b$ are $\sim 10.7 \mathrm{~m} \mathrm{~s}^{-1}$ (rms). The scatter is smaller (about $5.6 \mathrm{~m} \mathrm{~s}^{-1} \mathrm{rms}$ ) for spectra taken during the same night (generally over a few minutes). This last value seems then a lower limit for the internal errors in our bisector values for this star. In fact, one one hand we expect that real variations of the bisectors should show up over longer timescales, and on the other hand we can not exclude that other errors arise when comparing data from different nights, when e.g. a different wavelength calibration is used.

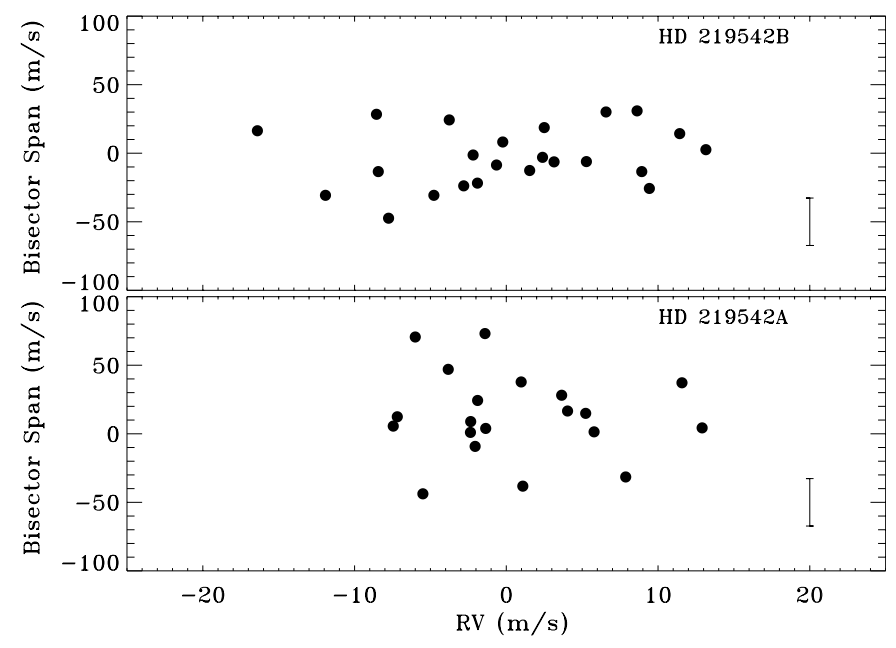

Fig. 14. Bisector span measurements vs. radial velocities for HD 219542 B (upper panel) and HD 219542 A (lower panel). There are not significant correlations for both stars. On the right corner the typical short term error on bisector span measurement is shown.

Hence, in the case of 51 Peg we may set an upper limit to real bisector span variations of about $9 \mathrm{~m} \mathrm{~s}^{-1}$ from our data. More stringent upper limit on the bisector span variations for $51 \mathrm{Peg}$ was obtained by Hatzes et al. $1999\left(\sim 7 \mathrm{~m} \mathrm{~s}^{-1}\right)$. However, the definition of the bisector span is different in that case, so that it is not easy to compare the two results. Transformation of our upper limit on bisector to a limit on the jitter in radial velocity is not obvious (it depends on the definition of the bisector span): however, in the case of HD 166435 (for which Queloz et al. 2001 used a definition similar to the present one) radial velocity variations are roughly 1.1 times the variations of the bisector spans. If the same rule then applies to our case, the upper limit to radial velocity jitter for $51 \mathrm{Peg}$ is $\sim 10 \mathrm{~m} \mathrm{~s}^{-1}$ : this is about 2.5 times larger than the limit obtained from photometric (Henry et al. 2000) and equivalent width variations (see below). However, we consider it as still an interesting upper limit.

In the case of HD 219542, the scatter of bisector spans is $31.2 \mathrm{~m} \mathrm{~s}^{-1}$ and $21.8 \mathrm{~m} \mathrm{~s}^{-1}$ for $\mathrm{A}$ and $\mathrm{B}$ component respectively. Bisector span is not significantly correlated with radial velocities for both stars (Fig. 14). Lower limits to measurement errors, determined as above from the results of consecutive spectra, is about $17.3 \mathrm{~m} \mathrm{~s}^{-1}$. Therefore the observed pattern can be explained mostly by measurement errors. Anyway, there is still room for bisector span variations corresponding to a radial velocity variations of $\sim 15 \mathrm{~m} \mathrm{~s}^{-1}$. This is larger than the observed scatter for HD $219542 \mathrm{~B}$, so that we can not exclude that the observed radial velocity variations are due to line asymmetries.

We note that while the bisector analysis is not conclusive in the present case, however it would be sufficient to disentangle the origin of radial velocity variations with amplitudes of a few tens of $\mathrm{m} \mathrm{s}^{-1}$. Finally, we note that a more detailed approach (that takes into account the instrumental line profile determined using the iodine lines) could be devised; however it requires a lengthy procedure and we postpone it to a forthcoming paper. 


\subsection{Limits on photometric variations}

The only photometric data set available suitable for a variability search is the Hipparcos photometry. However, it refers to the combined light of A and B components. When averaging data on a daily basis, the rms is $0.004 \mathrm{mag}$, without hints of variability above the measurement errors. No significant periodicities appeared applying the same procedures used in Sect. 5. The study of the Hyades by Paulson et al. (quoted by Henry et al. 2002) gives a relation between the radial velocity and photometric scatters of $\sim 3.3 \mathrm{~m} \mathrm{~s}^{-1} \mathrm{mmag}^{-1}$. Therefore, to justify the observed radial velocity dispersion, a photometric scatter 0.003 mag would be required. This is much lower than the limit placed by Hipparcos photometry, taking into account that HD 219542 B contributes only $40 \%$ of the composite light.

\subsection{Equivalent width variations}

We expect that activity may cause (small) variations in the average temperature in the line forming region. For this reason, we expect (small) variations in the average equivalent widths $(E W s)$ of spectral lines. This method is similar to the line depth ratio method considered by Gray (1994). We then selected among the line list used in Sect. 6.1 in the spectrum section clean of iodine lines the lines with large sensitivity to effective temperature changes, determined using appropriate spectral synthesis. The final selection includes about 40 lines for which variations of $1.0 \mathrm{~m} \AA$ in the $E W$ are due to temperature changes of less than $20 \mathrm{~K}$. A variations of $20 \mathrm{~K}$ in the stellar effective temperature causes a variation of about $0.017 \mathrm{mag}$ in stellar magnitude. We then measured the $E W$ s of the lines on all spectra of both components of HD 219542, as well as for the comparison star $51 \mathrm{Peg}$, using an our own purposely written code. On 51 Peg we found that for a single line, the typical rms scatter of the residuals of the $E W \mathrm{~s}$ with respect of the average value is $0.86 \mathrm{m \AA}$. The spectra have a typical $S / N$ of about 170 at this wavelength. Application of the formula by Cayrel (1988) would produce an expected error of $0.22 \mathrm{~m} \AA$ for these lines; however this formula neglects the contribution due to the continuum and background subtraction, that likely significantly increases the errors. Furthermore, internal errors obtained by considering the error for individual lines divided by the square root of the number of lines, likely underestimates the real error bar because they do not take into account correlation existing between nearby lines (due to positioning of the continuum level and errors in the background subtraction). We then measured the temperature variations properly weighting the lines according to their temperature sensitivity. The observed rms scatter of results for individual spectra is $2.5 \mathrm{~K}$, with internal errors $2.1 \mathrm{~K}$. This yields an upper limit to real spectrumto-spectrum of $1.3 \mathrm{~K}$. This on turn yields an upper limit to real variations in the luminosity of $51 \mathrm{Peg}$ of $0.0011 \mathrm{mag}$ (comparable to the results by Henry et al. 2000). This very strict upper limits constraints the jitter in radial velocity to be less than $3.6 \mathrm{~m} \mathrm{~s}^{-1}$ (using the quoted relation $3.3 \mathrm{~m} \mathrm{~s}^{-1} \mathrm{mmag}^{-1}$ ).

When the same procedure is applied to the spectra of HD 219542 B, we get much less stringent upper limits, due to the lower $S / N$ of the spectra. The observed rms of temperature is $8.34 \mathrm{~K}$; the internal error is $5.95 \mathrm{~K}$. This implies an upper limit to real variations in the effective temperature of $5.8 \mathrm{~K}$, and an upper limit to photometric variations of $0.0050 \mathrm{mag}$, about twice lower than the limit determined from the Hipparcos photometry. However, this limit is still large enough to accomodate a jitter in the radial velocity of about $15 \mathrm{~m} \mathrm{~s}^{-1}$, similar to the limit obtained from bisectors and well enough to justify observations.

We conclude that also this estimate of the expected jitter is not accurate enough for the present purpose.

\subsection{Expected radial velocity jitter}

The low chromospheric activity and the low rotational velocities $\left(R_{H K}=-5.36 \pm 0.13, v \sin i=1.9 \mathrm{~km} \mathrm{~s}^{-1}\right.$ for HD $219542 \mathrm{~A}$; $R_{H K}=-5.11 \pm 0.13, v \sin i=2.4 \mathrm{~km} \mathrm{~s}^{-1}$ for HD $219542 \mathrm{~B}$ ) indicate a low activity level. The jitter expected on the basis of the relation $\sigma_{v}$ vs. $v \sin i$ by Saar et al. (1998) is about $6 \mathrm{~m} \mathrm{~s}^{-1}$ and $8 \mathrm{~m} \mathrm{~s}^{-1}$ for HD $219542 \mathrm{~A}$ and B respectively. The $\log R_{H K}^{\prime}$ calibration gives instead $\sim 2$ and $3 \mathrm{~m} \mathrm{~s}^{-1}$ respectively. The calibration by Marcy (Marcy 2002, priv. comm.), that is based on a larger and higher precision data set than Saar et al. (1998) ones and takes into account the color dependence, predict instead a jitter of about 2 and $4 \mathrm{~m} \mathrm{~s}^{-1}$ for components $\mathrm{A}$ and $\mathrm{B}$ respectively.

To get a jitter of $8 \mathrm{~m} \mathrm{~s}^{-1}$ (required to explain the observed scatter), the chromospheric activity of HD 219542 B should be as high as $\log R_{H K}^{\prime} \sim-4.75$. Such a value seems to be excluded from the analysis presented in Sect. $2.3^{11}$.

The observed radial velocity jitter of HD $219542 \mathrm{~A}$ is very low, at most $4 \mathrm{~m} \mathrm{~s}^{-1}$, assuming that the $25 \%$ overestimate of errors of consecutive spectra holds also on longer time baseline. On the basis of the results by Paulson et al. (2002) for the Hyades, we do not expect that the colder component of a (coeval) pair shows larger jitter than the warmer one. However, the two stars may be on different phases of their activity cicle, so that the low jitter of HD 219542 A does not automatically guarantee the absence of moderate activity in its companion ${ }^{12}$.

Therefore, we can not exclude an activity origin for the velocity variations, taking into account that the line bisector, photometry and equivalent width analysis do not have the precision required to evidence the variations causing a $\sim 10 \mathrm{~m} \mathrm{~s}^{-1}$ radial velocity scatter.

\section{Limits on planetary companions}

As shown in Sect. 4.2, HD 219542 A does not show variations above errors and the statistical analysis did not reveal any significant periodicity in the velocities. The small velocity errors and the dense sampling of the observations allow us to derive firm upper limits for possible substellar companions. To this aim, we have developed a procedure that allow us to derive

\footnotetext{
11 Note that our $R_{H K}$ measurement was obtained in the season for which a larger radial velocity scatter is present.

12 If the planet around HD 219542 B does exist, the upper limit to the jitter estimated from residuals from the best fitting orbit, is $4.2 \mathrm{~m} \mathrm{~s}^{-1}$, similar to HD 219542 A.
} 
constraints for the masses of planets in circular as well as in eccentric orbit.

The method is a Monte-Carlo extraction and is as follows: we set a dense grid of mass and semimajor axis values covering the ranges $0.05-4 M_{J}$ and $0.01-3 \mathrm{AU}$. For each pair of mass and semimajor axis we randomly generated (with uniform distribution) 10000 combinations of orbital phase $T_{0}$ eccentricity $e$ and longitude of the periastron $\omega$ (the last two only for the eccentric case). We adopt the following ranges: $e<0.5625 \times$ $\log (P)-0.12$ for $P<80$ days (a conservative upper limit to the eccentricity of known exoplanets as a function of period) and $0 \leq e \leq 0.95$ for longer periods ${ }^{13} ; 0 \leq \omega \leq 360 \mathrm{deg}$; $0 \leq T_{0} \leq P_{\max }$.

For each set of orbital parameters, we create a synthetic radial velocity curve obtained by adding a Gaussian noise term (with the same rms as our $R V$-results) to a Keplerian orbit sampled at the epochs of our observations.

We then applied the F test, and computed the probability that the synthetic and the observed data sets have the same variance. If the probability is larger than $5 \%$ for more than $95 \%$ of the 10000 data sets for each mass-semimajor axis pair, than we took the planet with the corresponding mass and orbital semiaxis as compatible with the observed data. Otherwise, we considered the planet as excluded by our data, since its presence would have created a detectable excess of variability.

In this manner we obtain two regions in the masssemimajor axis plane the first where planets can be excluded, and an area below our limits (see Figs. 15, 16), where planets can still exist. It must be noted that the derived upper limits concern $m \sin i$.

Our method, based on the evaluation of the excess of radial velocity variability caused by the presence of hypothetical planets, allows a complete exploration of the possible orbital parameters for eccentric orbits (the real case, since most of the known planets are in eccentric orbits). This is not feasible when the synthetic data are examined using methods like the periodogram analysis (see Endl et al. 2002).

Note that this procedure attempts to answers the question: would the presence of a planet more massive than this limit at the given separation have caused scatter incompatible with observed data? This is a somewhat different question from that answered by the usual procedure, that consider the detection of a planet with a high level of confidence (typically 99\%) using procedures based on the periodogram analysis (see e.g. Endl et al. 2002; Cumming et al. 1999). These authors formulate the problem slightly differently: they determine the minimum amplitudes for planetary signal at various separations, which can be detected by their data at a statistical significance of $>99 \%$. The two approaches are rather different, because in principle, a planet could be present even if its detection is not significant at a high level of confidence. However, we repeated our procedure for the case of $\alpha$ Cen A, for which upper limits to planet masses have been obtained by Endl et al. (2001) using the alternative approach, and we find that our procedure (when

13 This upper limit is justified on the basis on the detection of a planet with $e=0.93$ (HD80606b, Naef et al. 2001).

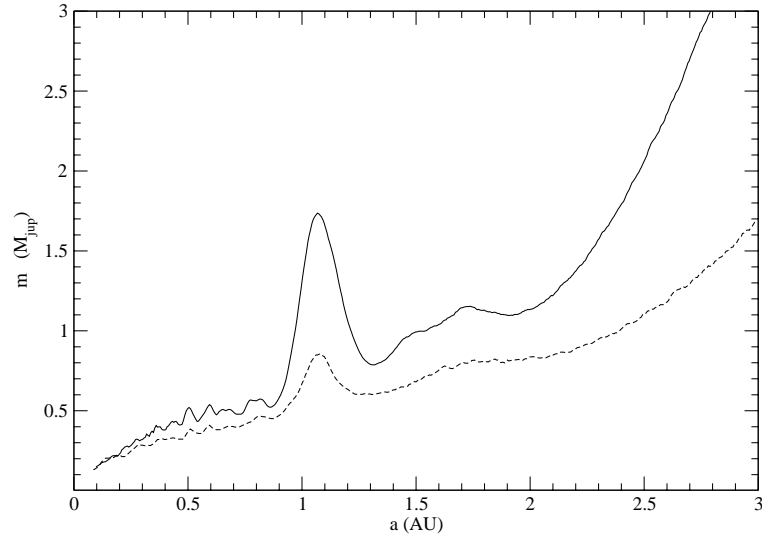

Fig. 15. Limits on planets around HD 219542 A in circular (dashed line) and eccentric (continuous line) orbits derived using the Monte Carlo procedure described in the text.

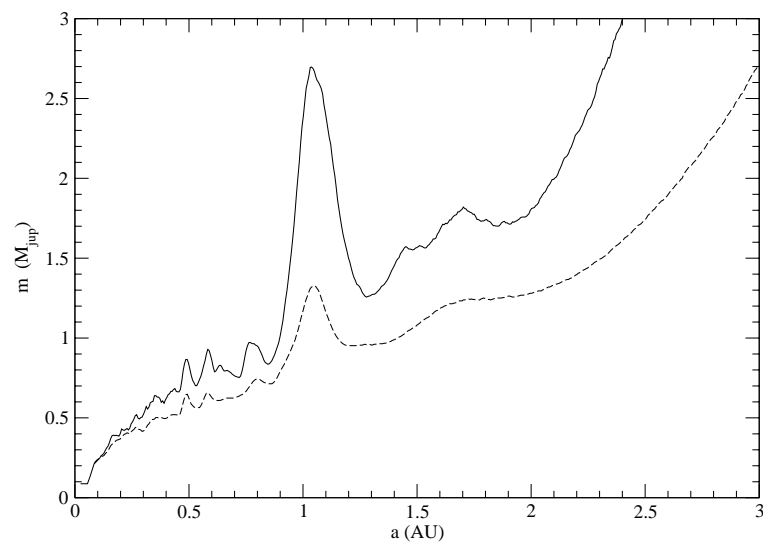

Fig. 16. Limits on planets around HD 219542 B in circular (dashed line) and eccentric (continuous line) orbits derived using the Monte Carlo procedure described in the text.

assuming circular orbits) closely reproduces the same upper limits to planetary masses obtained in that paper.

Figure 15 shows the results for HD 219542 A. The limits for planets in eccentric orbit are less stringent than for planets in circular orbits, becuase of the flexibility of shape of the Keplerian curve for high eccentricties. Planets with $m \sin i>2 M_{J}$ are excluded for separation smaller than $2.4 \mathrm{AU}$ also 51 Peg-like planets with masses $m \sin i>0.15 M_{J}$ are excluded. For orbital periods exceeding the baseline of our measurement the constraint becomes shallower. A peak corresponding to the 1 year observing window can be noticed, especially in the eccentric case. Figure 16 shows the results for HD $219542 \mathrm{~B}$. The limits are less stringent because of the larger scatter of the velocities.

\section{Discussion}

The main results of this paper are the following:

1. we detected a low-amplitude RV variation for HD 219542 B which could be due to a Saturn mass planet in close orbit; 
2. for component $\mathrm{A}$, the star which shows signs of planetary accretion, we can place stringent upper limits on the existence of orbiting giant planets.

Taking into account the possibility that the observed radial velocity variation of component B is caused by modest stellar activity, we think that a lengthy discussion on the implications of the presence of a planet around HD $219542 \mathrm{~B}$ is premature.

However, we note that HD $219542 \mathrm{Bb}$, if it exists, should represent the third case of a planet in a binary system around the lithium poor component of the pair, after $16 \mathrm{Cyg} \mathrm{B}$ and HD 178911. In the case of HD 219542 and $16 \mathrm{Cyg}^{14}$, the star without detected planet shows also the signatures of accretion of planetary material. In these cases, the signatures of accretion might be anti-correlated with the presence of planets.

HD 219542 B is by itself a metal-rich star, with metallicity typical for the stars with planets $([\mathrm{Fe} / \mathrm{H}]=+0.20)$. If we take the abundances of HD $219542 \mathrm{~B}$ as the original values one for the system (i.e. assuming that no enrichment occurred for HD 219542 B) it implies that the system was originally very metal rich. This might suggest that accretion events are more likely on very metal rich stars, possibly because they form more planets and/or they have more massive disks. The full analysis of the SARG sample would tell us if such enrichment events are limited to very metal rich stars or they can happen also for metal poor stars.

The lack of giant planets in close orbit around HD 219542 A leaves several possibilities for the origin of the observed signatures of accretion open.

- Planet formation did not occur around HD 219542 A and a significant portion of the circumstellar disk fell into the stars possibly because of the dynamical perturbation of HD 219542 B. This possibility is neither excluded nor supported by the possible stellar orbital parameters given in Sect. 3. If the planet around component B is confirmed, it would indicate that gravitational influence did not disrupt the planet formation process.

- One or more giant planets orbiting around HD 219542 A were scattered toward the star by other planets in the system (Marzari \& Weidenschilling 2002) or by the perturbation of HD 219542 B. In the first case, we would expect to find a planet in an external eccentric orbit. The continuation of our radial velocity monitoring could reveal that.

- No giant planets formed in the system while terrestrial ones formed in the inner regions. Some of them may have been scattered into the star by mutual interactions.

Our results exclude instead a scenario with a giant planet that formed near the ice boundary (4-5 AU) and migrated inward, causing the infall on the star of the inner part of the disk and/or terrestrial planets already formed (we would have detected such planet, unless the planet itself was engulfed by the star).

The study of a few pairs clearly does not allow us to reach a firm conclusion about the frequency of pairs with chemical

\footnotetext{
14 To our knowledge, a high precision differential chemical composition analysis was not performed for HD 178911, but this would be complicated by the double line spectroscopic binarity of the primary.
}

composition differences; a general picture is missing. How frequent are these pairs? Is there any link between the pollution phenomena and some properties like the physical separation of the components and the initial composition? How frequent are planets around the components of nearly equal mass binaries with separation 200-300 AU and what are their orbital characteristics? Are planets really missing around polluted stars?

Our on-going survey including a sample of about 50 binaries might allow some deeper insight into these very exciting topics.

Acknowledgements. We warmly thank A. Bragaglia, F. Leone, S. Randich, F. Sabbadin, and P. Sestito for taking some spectra during their observing time. We thank the TNG staff for its help in the observations. We are grateful to R. E. M. Griffin for supplying the Orbitsolver code. We are grateful to M. Kürster and S. Els for useful insights on the radial velocity measurements and to G. Marcy for providing us its jitter calibration. We thank the referee A. Hatzes for a very encouraging report. This publication makes use of public data obtained during FEROS Commissioning and of data products from the Two Micron All Sky Survey, which is a joint project of the University of Massachusetts and the Infrared Processing and Analysis Center/California Institute of Technology, funded by the National Aeronautics and Space Administration and the National Science Foundation. This research has made use of the SIMBAD database, operated at CDS, Strasbourg, France. ME acknowledges support from NSF Grant AST-9808980 and NASA Grant NAG5-9227.

\section{References}

Aitken, R. G. 1932, New general catalogue of double stars within 120 of the North pole, Carnegie Inst. Washington Pub. No. 417

Baliunas, S. L., Donhaue, R. A., Soon, W. H., et al. 1995, ApJ, 438, 269

Barbieri, M., \& Gratton, R. 2002, A\&A, 384, 879

Bouvier, J. 1990, AJ, 99, 946

Butler, R. P., Marcy, G. W., Vogt, S. S., et al. 2002, ApJ, 578, 565

Butler, R. P., Marcy, G. W., Vogt, S. S., et al. 2003, ApJ, 582, 455

Cayrel, G. 1988, in The Impact of Very High S/N Spectroscopy on Stellar Physics, IAU Symp., 132, 345

Cochran, W. D., Hatzes, A. P., Butler, R. P., \& Marcy, G. W. 1997, ApJ, 483, 457

Corbally, C. J., \& Garrison, R. F. 1980, PASP, 92, 493

Cuypers, J., \& Seggewiss, W. 1999, A\&AS, 139, 425

Cumming, A., Marcy, G. W., \& Butler, R. P. 1999, ApJ, 526, 890

Desidera, S. 2000, Ph.D. Thesis, University of Padova

Eggen, O. J. 1960, MNRAS, 120, 563

Endl, M., Kürster, M., \& Els, S. 2000, A\&A, 362, 585

Endl, M., Kürster, M., Els, S., Hatzes, A. P., \& Cochran, W. D. 2001, A\&A, 374, 675

Endl, M., Kürster, M., Els, S., et al. 2002, A\&A, 392, 671

ESA 1997, The Hipparcos and Tycho Catalogues, ESA SP-1200

Fischer, D., Marcy, G. W., Butler, R. P., et al. 2001, ApJ, 551, 1107

Fischer, D., Marcy G. W., Butler, R. P., Laughlin, G., \& Vogt, S. S. 2002, ApJ, 564, 1028

Girardi, L., Bressan, A., Bertelli, G., \& Chiosi, C. 2000, A\&AS, 141, 371

Glebocki, R., Musielak, G., \& Stawikowski, A. 1980, Acta Astron., 30,453

Gonzalez, G. 1997, MNRAS, 285, 403

Gonzalez, G., Laws, C., Tyagi, S., \& Reddy, B. E. 2001, AJ, 121, 432

Gratton, R. G., Bonanno, G., Bruno, P., et al. 2001a, Exp. Astron., 12, 107 
Gratton, R. G., Bonanno, G., Claudi, R. U., et al. 2001b, A\&A, 377, 123 (Paper I)

Gratton, R. G., Carretta, E., Claudi, R. U., et al. 2002, in Scientific Frontiers in Research on Extrasolar Planets, ed. D. Deming, ASP Conf. Ser., in press

Gray, D. F. 1992, The Observations and Analysis of Stellar Photospheres (Cambridge University Press)

Gray, D. F. 1994, PASP, 106, 1248

Gray, D. F. 1997, Nature, 385, 795

Griffin, R. F., \& Cornell, A. P. 1998, MNRAS, 293, 151

Hale, A. 1994, AJ, 107, 306

Hasinger, G., Burg, R., Giacconi, R., et al. 1993, A\&A, 275, 1

Hatzes, A. P., Cochran, W. C., \& Bakker, E. J. 1998, Nature, 391, 154

Hatzes, A. P., Cochran, W. D., Endl, M., et al. 2003, ApJ, submitted [astro-ph/0305110]

Hauser, H. M., \& Marcy, G. W. 1999, PASP, 111, 321

Heintz, W. D. 1978, Double Stars (Dordrecht: D. Reidel Publishing Company)

Henry, G. W., Baliunas, S. L., Donahue, R. A., Fekel, F. C., \& Soon W. 2000, ApJ, 531, 415

Henry, G. W., Donahue, R. A., \& Baliunas, S. L. 2002, ApJ, 577, L111

Henry, T. J., Soderblom, D. R., Donhaue, R. A., \& Baliunas, S. L. 1996, AJ, 111, 439

Holden, F. 1977, PASP, 89, 588

Holman, M., Touma, J., \& Tremaine, S. 1997, Nature, 386, 254

Holman, P. A., \& Weigert, M. J. 1999, AJ, 117, 621

Hünsch, M., Schmitt, J. H. M. M., Sterzik, M. F., \& Voges, W. 1999, A\&AS, 135, 319

Kaufer, A., Stahl, O., Tubbesing, S., et al. 1999, ESO Messenger, 95

King, J. R., Delyannis, C. P., Hiltgen, D. D., et al. 1997, AJ, 113, 1871

Kürster, M., Schmitt, J. H. M. M., Cutispoto, G., \& Dennerl, K. 1997, A\&A, 320, 831

Israelian, G., Santos, N. C., Mayor, M., \& Rebolo, R. 2001, Nature, 411,163

Laws, C., \& Gonzalez, G. 2001, ApJ, 533, 405

Lomb, N. R. 1976, Ap\&SS, 39, 477

Marcy, G. W., Butler, R. P., \& Vogt, S. S. 2000, ApJ, 536, L43

Marcy, G. W., Butler, R. P., Fischer, D., et al. 2002, ApJ, 581, 1375

Marzari, F., \& Weidenschilling, S. J. 2002, Icarus, 156, 570

Mazeh, T., Krymolowsky, Y., \& Rosenfeld, G. 1997, ApJ, 477, L103

Murdoch, K. A., Hearnshaw, J. B., \& Clark, M. 1993, ApJ, 413, 349
Murray, N., Chaboyer, B., Arras, P., Hansen, B., \& Noyes, R. W. 2001, ApJ, 555, 801

Naef, D., Latham, D. W., Mayor, M., et al. 2001, A\&A, 375, L27

Nidever, D. L., Marcy, G. W., Butler, R. P., Fischer, D. A., \& Vogt, S. S. 2002, A\&AS, 141, 503

Olsen, E. H. 1998, A\&AS, 129, 431

Paulson, D. B., Saar, S. H., Cochran, W. D., \& Hatzes, A. P. 2002, AJ, 124,572

Perryman, M. A. C., et al. 1998, A\&A, 331, 81

Pinsonneault, M. H., De Poy, D. L., \& Cofee, M. 2001, ApJ, 556, L59

Popovic, G. M., \& Pavlovic, R. 1997, Bull. Astron. Beograd, 155, 97

Queloz, D., Henry, G. W., Sivan, J. P., et al. 2001, A\&A, 379, 279

Randich, S. 2000, in Stellar Clusters and Associations: Convection, Rotation, and Dynamos, ed. R. Pallavicini, G. Micela, \& S. Sciortino, ASP Conf. Ser., 198, 401

Reddy, B. E., Lambert, D. L., Laws, C., Gonzalez, G., \& Covey, K. 2002, MNRAS, 335, 1005

Ryan, S. G. 2000, MNRAS, 316, L35

Saar, S. H., Butler, R. P., \& Marcy, G. W. 1998, ApJ, 498, L153

Sandquist, E. L., Dokter, J. J., Lin, D. N. C., \& Mardling, R. A. 2002, ApJ, 572, 1012

Santos, N. C., Israelian, G., \& Mayor, M. 2001, A\&A, 373, 1019

Sasselov, D. 2002, in Scientific Frontiers in Research on Extrasolar Planets, ed. D. Deming, ASP Conf. Ser., in press

Scargle, J. D. 1982, ApJ, 263, 835

Smith, V. V., Cunha, K., \& Lazzaro, D. 2001, AJ, 121, 3207

Taylor, B. J. 2000, A\&A, 362, 563

Thorburn, J. A., Hobbs, L. M., Delyannis, C. P., \& Pinsonneault, M. H. 1993, ApJ, 415, 150

Udry, S., Mayor, M., Queloz, D., et al. 2002, in Scientific Frontiers in Research on Extrasolar Planets, ed. D. Deming, ASP Conf. Ser., in press

van Albada-van Dien, E. 1978, A\&AS, 31, 353

van Albada-van Dien, E. 1983, A\&AS, 52, 193

Voges, W., Aschenbach, B., Boller, T., et al. 2000, ROSAT AllSky Survey Faint Source Catalog, Max Plank Institut fur Extraterresstriche Physik, Garching

Wilson, R. E. 1953, General Catalogue of Stellar Radial Velocities, Carnegie Inst. Washington D.C. Publ., 601

Zucker, S., Naef, D., Latham, D. W., et al. 2002, ApJ, 568, 363

Zucker, S., \& Mazeh, T. 2002, ApJ, 568, L113 